\title{
Theory of the Helix-Coil Transition in Singly Cross-Linked, Two-Chain, Coiled Coils
}

\author{
Jeffrey Skolnick ${ }^{\dagger}$ \\ Department of Chemistry, Washington University, St. Louis, Missouri 63130. \\ Received November 9, 1984
}

\begin{abstract}
Two models of the helix-coil transition in singly cross-linked, two-chain, coiled coils are presented. In the more restrictive and simpler, loops-excluded model, interior random coil loops between interacting pairs of helices are a priori excluded as was done for non-cross-linked chains. In the more general interior eyelet model, in addition to those states allowed in the loops-excluded model, constrained interior random coil loops can originate at the cross-link and terminate on the interacting pair of helices; such loops of course pay a heavy entropic price. Expressions for each model, in the perfect-matching limit, are presented for the partition function, the overall helix content $f_{\text {he }}$, the helix probability profiles, and the fraction of random coils in end random coil sequences. The formalism is applicable to any singly cross-linked, two-chain, coiled coil of arbitrary amino acid sequence and incorporates into the theory the quasi-repeating heptet nature of the primary sequence of the two-chain, coiled coil tropomyosin. Application of the theory to homopolymers revealed that the interior eyelet model gives results essentially identical with those of the loops-excluded model; thus constrained interior random coil loops including the cross-link are statistically unimportant, and conformations containing the single interacting helical stretch always include the cross-linked pair of blocks. The dependence of $f_{\mathrm{he}}$ on the location of the cross-link, on chain length, on the helix-helix interaction parameter $w$, on the Zimm-Bragg intrachain helix initiation parameter $\sigma$, and on the effective interchain helix initiation parameter $r_{\phi}$ are investigated.
\end{abstract}

\section{Introduction}

Over the past several years, we have developed a theory of the helix-coil transition in non-cross-linked, two-chain, coiled coils. ${ }^{1-4}$ Two-chain, coiled coils are a particularly simple model system for elucidating some aspects of the more general protein-folding problem. In the limit of high helix content, they consist of two parallel, $\alpha$-helical chains with a slight supertwist. ${ }^{5-12}$ Thus their secondary structure is an $\alpha$-helix, there is no tertiary structure, and the quaternary structure consists of the side-by-side association of the two supercoiled, $\alpha$-helical chains. Hence because of their structural simplicity they are amenable to treatment by a statistical mechanical model. The theory is very much in the spirit of that of Zimm and Bragg for isolated single chains ${ }^{13}$ and appends to the intrachain helix initiation and propagation parameters, $\sigma$ and $s$, two interchain parameters, a helix-helix interaction parameter $w(-R T$ ln $w$ is the free energy of a positionally fixed, interacting pair of $\alpha$-helical turns (blocks) relative to the case where the two helical turns are positionally fixed but noninteracting) and an interchain helix initiation parameter, $q_{\phi}$, equal to the effective volume of configurational space accessible to the center of mass of an $\alpha$-helical block in one of the chains when the other $\alpha$-helical block is held fixed. The theory has been extended to include both the effects of loop entropy and mismatch ${ }^{2-4}$ in the loops-excluded, imperfect-matching (LEIM) model. More recently, the LEIM model has been successfully applied to rabbit $\alpha$ tropomyosin. ${ }^{14}$ A single algorithm for $w(T)$ extracted from circular dichroism data is found to fit reasonably well the experimentally determined helix content vs. temperature curves at near-neutral and acidic $\mathrm{pH}$, is consistent with the experimentally measured weight fraction of single chains vs. temperature. ${ }^{15}$ and is consistent with the fraction of chains that can be cross-linked at low temperature and at near-neutral $\mathrm{pH} .{ }^{16}$ Based on the success of the theory, as applied to non-cross-linked chains, in the present paper we extend the theory to chains containing a single crosslink.

In previous work on non-cross-linked chains of moderate length, we demonstrated the net effect of loop entropy is

${ }^{\dagger}$ Alfred P. Sloan Foundation Fellow. to prohibit interior random coil states between stretches of interacting pairs of helices. ${ }^{2}$ In the spirit of previous work, we develop the "loops-excluded" model of two-chain, coiled coils in which we a priori set equal to zero the statistical weights of all interior random coil stretches originating at the cross-link and terminating on an interacting pair of helices. The presence of the fully random coil state at the cross-linked block $N_{\mathrm{C}}$ and to the right of the cross-linked block implies that any helical states in blocks $i>N_{\mathrm{C}}$ cannot be interacting helical states. Similarly if block $i<N_{\mathrm{C}}$ is a random coil state, then it must have been preceded by noninteracting helical or random coil states.

The second less restrictive model we develop is the "interior eyelet" model, where we permit random coiled loops originating at the cross-link to occur and explicitly account for the statistical weight of such closed random coil loops by a generalization of the loop entropy expression developed by Schellman, ${ }^{17}$ Flory, ${ }^{18}$ and Jacobson and Stockmayer. ${ }^{19}$ In particular, two kinds of random coil loops or eyelets originating at the cross-link are allowed. There are type I eyelets, in which both chains from the cross-link to the interfacial pair of helical states in block $i$ are randomly coiled, and type II eyelets, in which one chain contains a completely helical stretch propagating from the interacting pair of helices to the cross-link and the other chain is in a completely random coil state from the cross-link to the block next to the interfacial interacting helix-coil state. Thus, there may be an interacting pair of helices to the left and/or right of the cross-link, interrupted by closed interior random coil loops originating at the cross-link. Moreover, on the basis of previous work on the relative importance of interior random coil loops between interacting helices, ${ }^{2}$ we a priori exclude such interior loops if the cross-link is not involved. We would expect closed interior loops involving the cross-link to be most important if the cross-link is close to a chain end but they should contribute negligibly if the cross-link is located near the middle of a chain. In the latter case the results obtained from the interior eyelet model should reduce to the far simpler loops-excluded model. Actually, as we demonstrate below, for chains of moderate length, the effect of loop entropy is so prohibitive as to essentially entirely eliminate the interior random coil loops originating at the cross-link. 
To account for the geometric constraints imposed by the fixed bond lengths and bond angles of a real helix in the interior eyelet model, we assume that residues in the cross-linked block and part of a type I or II random coil loop are completely randomly coiled. As mentioned above, even by assuming the least restrictive case, that is, that type I or II random coils are Gaussian chains, the interior eyelet model gives results essentially identical with those of the loops-excluded model; hence this assumption as a matter of practice is really not required.

To incorporate the quasi-repeating heptet nature of the primary sequence designated by the letters $a-g$ of the two-chain, coiled coil tropomyosin ${ }^{20-22}$ into the theory, we have (as was previously done ${ }^{1-4}$ ) divided the chain into blocks, the $i$ th block of which contains $m_{i}$ residues (in tropomyosin $m_{i}=4$ and $m_{i+1}=3$ ) and employ the coarse-graining method originally developed for single chains by Crothers and Kallenbach ${ }^{23}$ and conveniently reformulated by Poland and Scheraga. ${ }^{24}$ We remind the reader that in tropomyosin positions a and $d$ tend to be occupied by hydrophobic residues and positions e and $g$ by anionic and cationic residues, respectively. We use coarse graining not as an approximation but rather as a statement of the physics that in order to have interhelical interactions all the residues in hydrophobic-type blocks (a-d) in both chains (or all the residues in the charge-type blocks containing salt bridge forming residues $e$ and g) must be in the helical state.

In previous work, Mattice and Skolnick (MS) ${ }^{25}$ have applied the " $3 \times 3$ " forward and backward looking matrix technique ${ }^{26}$ that tries to better account for end effects to the helix-coil transition of singly cross-linked, two-chain, coiled coils. This work was subsequently applied to tropomyosin by Maroun and Mattice (MM). ${ }^{27}$ The loopsexcluded model developed here with $m=1$ is the " $2 \times 2$ " matrix analogue, in which end effects are assigned à la Zimm and Bragg to one of the ends of the helical stretch. For a homopolymer, the two methods give identical results, but for a heteropolymer the results based on the singlechain " $3 \times 3$ " matrix give different answers than that based on the single-chain " $2 \times 2$ " matrix. In some sense the " 3 $\times 3$ " matrix method may be preferable in that it gives the same answer no matter which end is chosen-but this may not necessarily mimic the physical situation, as some recent work by Ihara, Ooi, and Takahashi indicates. ${ }^{28}$

There are however several problems with the previous work on cross-linked two-chain, coiled coils that the present treatment addresses and remedies. First of all, unlike the MS model, in both the loops-excluded and interior eyelet models, we consider the quasi-repeating heptet explicitly rather than invoke the incorrect $m=1$ approximation that ignores this fundamental aspect of the physics. Second, even if $w$ is normalized to the value per pair of residues, one cannot apply values of the helix-helix interaction parameter $w$ obtained for non-cross-linked chains and based on the " $2 \times 2$ " matrix method to crosslinked chains based on the " $3 \times 3$ " matrix method as was done by Maroun and Mattice. The helix content of an isolated single chain of tropomyosin calculated via the former technique is about $14 \%$ whereas by the latter technique it is $19 \% .^{26}$ This difference in intrinsic stability is substantially magnified by values of the helix-helix interaction parameter that are greater than one. Thus, if a " $3 \times 3$ " matrix method fit to cross-linked data is to be done, it should be based on the $w$ extracted from a fit using the " $3 \times 3$ " matrix method, as applied to non-cross-linked chains. Third, the definition of the parameter $\omega$ introduced by MS to reflect the difficulty in aligning the helices at the cross-link is somewhat incorrect. It is not as MM indicate the ratio of the probabilities, calculated in a rotational isomeric approximation, of the cross-linked configurations that are consistent with the interacting helical conformation to the probability of these states in noninteracting conformations. Rather, following Mayer and Mayer ${ }^{29}$ it is the ratio of the effective volume $u_{\phi}$ accessible to one of the $\alpha$-helical turns when the other interacting $\alpha$-helical turn is held fixed to the effective volume $v_{\phi}$ accessible to one of the cross-linked blocks in any noninteracting configuration when the other cross-linked block is held fixed and includes the configurational entropy of the cross-link itself. Finally, the "interior eyelet" model by permitting type I and II interior random coil states allows us to vary the location of the cross-link. Thus, we can treat two-chain, coiled coils that are cross-linked near the chain ends, as is the case in a synthetic analogue of tropomyosin. ${ }^{30}$ In the loops-excluded and MS models, there is always the concern that types I and II random coil states might be important for two-chain, coiled coils containing a cross-link near the chain ends. However, the loops-excluded and MS models have no means of assessing the actual importance of such states, as they assume such random coil loops have zero statistical weight.

The organization of this paper is as follows. In section IIA we flesh out the description of the general features of the loops-excluded and interior eyelet models of the helix-coil transition in cross-linked, two-chain, coiled coils. In sections IIB and IIC, we develop the loops-excluded and interior eyelet models, respectively. For each model, expressions are presented for the partition function, the overall helix content, the helix probability profiles, and the fraction of random coils in end random coil sequences for a heteropolymeric, two-chain, coiled coil containing a single cross-link. Section III presents calculations on a hypothetical homopolymeric, two-chain, coiled coil designed to illustrate the qualitative effects of the cross-link on the character of the helix-coil transition and compares results obtained via the loops-excluded model with the more general interior eyelet model. Finally, section IV gives an overview of the present paper and its conclusions and suggests several possible directions of future research.

\section{Theory}

A. General Considerations. This section develops the necessary theoretical formalism to treat the helix-coil transition in a heteropolymeric, two-chain, coiled coil containing a single cross-link. For convenience, we assume both chains are identical. Let each chain contain $N_{\mathrm{B}}$ blocks, in which the $i$ th block contains $m_{i}$ residues and in which the first amino acid residue in block one is the $\mathrm{N}$-terminal residue and the last amino acid residue in block $N_{\text {B }}$ is the C-terminal residue. The $\mathrm{N}$ terminus ( $\mathrm{C}$ terminus) shall be referred to as the left (right) part of the molecule. For the development presented below, we assume that both chains are cross-linked at block $N_{\mathrm{C}}$ and shall neglect the possibility of out-of-register conformations of the two chains. The more general case, including the possibility of out-of-register conformations will be treated in a future publication. We consider the simplest, inregister, case below.

In the $N_{\mathrm{C}}$ th block there are $m_{l}$ residues to the left of and including the cross-linked residue, and $m_{r}$ residues to the right of and including the cross-link; hence $m_{N_{\mathrm{C}}}=m_{l}+$ $m_{r}-1$. Let $l_{c}$ be the ratio of the length of the cross-link to the virtual bond length $\left(=3.80 \AA^{31}\right)$ of an amino acid residue.

In what follows, we shall require the statistical weights of conformations associated with blocks not at the cross- 
link and not in interior random coil loops. Denote $\mathrm{H}(\mathrm{C})$ as a helical (random coil) block, that is a state where all $m_{i}$ residues are helical (randomly coiled), and $[\mathrm{H}] \mathrm{C}(\mathrm{C}[\mathrm{H}])$ an interfacial helix-coil (coil-helix) block. In an isolated single-chain poly(amino acid), the statistical weights of the allowed conformations for block " $i$ " are

$$
\begin{array}{cl}
\text { conformation } & \text { statistical weight } \\
\text { [C]C } & 1 \\
{[\mathrm{C}] \mathrm{H}} & \tau_{i}=\sum_{j=1}^{m_{i}} \sigma_{j} \prod_{k=j}^{m_{i}} s_{k} \\
{[\mathrm{H}] \mathrm{C}} & \mathcal{s}_{i}=1+\sum_{j=1}^{m_{i}-1} \prod_{k=1} s_{k} \\
{[\mathrm{H}] \mathrm{H}} & \mathrm{SM}_{i}=\prod_{j=1}^{m_{j}} s_{j}
\end{array}
$$

For the pair of cross-linked but noninteracting chains, $\sigma$ and $s$ are taken to be the same as in the non-cross-linked chain. We also need an additional parameter $v_{\phi}$, equal to the volume of configurational space available to the center of mass of block $N_{\mathrm{C}}$ in one of the two chains when block $N_{\mathrm{C}}$ in the other chain is held fixed, times the ratio of the volume of configuration space spanned by the internal degrees of freedom of the cross-linked to the non-crosslinked block pair $N_{\mathrm{C}}$. Observe that $v_{\phi}$ contains the contribution of the all-random-coil state as well as the $\alpha$-helical state of each of the cross-linked blocks.

For the chains containing at least one interacting helical block pair, we require the two additional quantities $w_{i}$ and $u_{\phi i}$ that are in principle site dependent. $w_{i}$ is the interchain helix propagation parameter. $u_{\phi i}$ behaves like an interchain initiation parameter and is the product of three factors: (a) the volume of configurational space accessible to the center of mass of the $i$ th block in one of the chains when the other $i$ th block is fixed and includes the configurational entropy of the side chains involved in the cross-link, (b) the ratio of the volume of configurational space spanned by the internal degrees of freedom in the pair of interacting blocks to that in the pair of noninteracting blocks, and (c) the ratio of the allowed relative angular orientation of the two $\alpha$-helical turns in the interacting pair of blocks to $4 \pi$. In what follows for convenience we shall assume a site-independent $u_{\phi i}$ and $w_{i}$. In principle, these can be extracted from experiment.

B. Loops-Excluded Model of Singly Cross-Linked, Two-Chain, Coiled Coils. Construction of the Partition Function. In the case of a two-chain, coiled coil containing a single interacting helical stretch, the partition function is given by

$$
\begin{gathered}
Z_{\mathrm{d}}{ }^{0}=v_{\phi} Z_{\mathrm{m}}{ }^{2}+u_{\phi} \mathbf{J} * \prod_{i=1}^{N_{\mathrm{B}}} \mathbf{U}_{\mathrm{si}} \mathbf{J} \\
Z_{\mathrm{d}}{ }^{0}=v_{\phi} Z_{\mathrm{c}}{ }^{0}=v_{\phi} Z_{\mathrm{m}}{ }^{2}+u_{\phi} Z_{\mathrm{int}}
\end{gathered}
$$

in which $Z_{m}$ is the internal partition function of an isolated single chain. $v_{\phi}$ has been defined following eq II-1. In the following, a superscript zero will label the quantity calculated via the loops-excluded model.

$$
Z_{\mathrm{m}}=\mathbf{J}_{2} * \prod_{i=1}^{N_{\mathrm{B}}} \mathbf{U}_{\mathrm{m} i} \mathbf{J}_{2}
$$

in which $\mathbf{J}_{2}{ }^{*}=\operatorname{row}(1,0), \mathbf{J}_{2}=\operatorname{col}(1,1)$, and

$$
\mathrm{U}_{\mathrm{m} i}=\left[\begin{array}{ll}
1 & \tau \\
\mathfrak{S} & \mathrm{SM}
\end{array}\right]_{i}
$$

$Z_{\text {int }}$ contains the contribution to the partition function of the interacting helical states and $u_{\phi}$ has been defined above. $\mathbf{J} *$ is a row vector of dimension 12 and consists of unity followed by 11 zeros. $\mathbf{J}$ is a column vector of dimension 12 and consists of four zeros followed by eight ones. If $i<N_{\mathrm{C}}, \mathbf{U}_{\mathrm{s} i}$ is a $12 \times 12$ matrix of the form

$$
\mathrm{U}_{\mathrm{si}}=\left[\begin{array}{lll}
\mathrm{U}_{\mathrm{d}} & \mathrm{U}_{\mathrm{CH}} & 0_{4} \\
\mathbf{0}_{4} & \mathrm{U}_{\mathrm{HH}} & 0_{4} \\
\mathbf{0}_{4} & \mathbf{0}_{4} & 0_{4}
\end{array}\right] i
$$

wherein $\mathrm{O}_{4}$ is a $4 \times 4$ null matrix (in the following we shall employ the convention that $\mathbf{O}_{n}$ is an $n \times n$ null matrix).

$$
\mathbf{U}_{\mathrm{d}}=\mathbf{U}_{\mathrm{m} 1} \otimes \mathrm{U}_{\mathrm{m} 2}
$$

Subscripts 1 and 2 refer to chains one and two, respectively. " $\otimes$ " represents the direct product, and

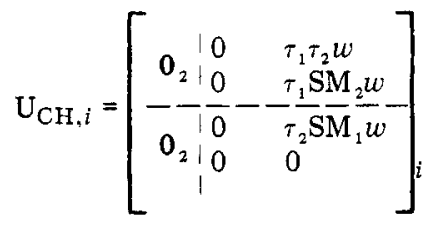

is the standard "initiating" matrix of the interaction helical stretch and

$$
\mathrm{U}_{\mathrm{HH}, i}=\left[\begin{array}{c:cc}
\mathbf{0}_{2} & \mathbf{0}_{2} \\
\hdashline \mathbf{0}_{2} & 0 & 0 \\
& 0 & \mathrm{SM}_{1} \mathrm{SM}_{2} w
\end{array}\right] i
$$

is the standard propagating matrix of the interacting helical stretch. $T$ and SM may be found in eq II-1.

Equation II-4a represents the fact that block $i$ with $i<$ $N_{\mathrm{C}}$ can be in a noninteracting state to the left of the interacting pair of $\alpha$-helices or may be part of the interacting helical stretch that will propagate through the cross-link. Thus eq II-4a specifies that there are no closed interior random coil loops to the left of and including the cross-link. If $i=N_{\mathrm{C}}$

$$
\mathrm{U}_{s i}=\left[\begin{array}{lll}
\mathrm{U}_{\mathrm{d}} & \mathrm{U}_{\mathrm{CH}, N_{\mathrm{C}}} & \mathbf{0}_{4} \\
\mathbf{0}_{4} & \mathrm{U}_{\mathrm{HH}} & \mathbf{0}_{4} \\
\mathbf{0}_{4} & \mathbf{0}_{4} & \mathbf{0}_{4}
\end{array}\right]_{N_{\mathrm{C}}}
$$

wherein $\mathbf{U}_{\mathrm{d}}$ is defined in eq II- $4 \mathrm{~b} ; \mathbf{U}_{\mathrm{CH}, N_{\mathrm{C}}}$ is of the identical form as eq II-4c, but here we replace $\tau$ defined in eq II-1 by

$$
\tau_{N_{\mathrm{C}}}=\sum_{j=1}^{m_{l}} \sigma_{j} \prod_{k=1}^{m_{N \mathrm{C}}} s_{k}
$$

That is, if block $N_{\mathrm{C}}$ is a [C]H (initiating) type state and if closed random coil loops are excluded, then residues $m_{l}$ +1 to $m_{N_{\mathrm{C}}}$ must be helical; otherwise there will be a closed interior random coil loop to the right of and including the cross-link. $\mathbf{U}_{\mathrm{HH}, i}$ is defined in eq II-4d with $i=N_{\mathrm{C}}$. In eq II-5a, $\mathbf{U}_{\mathrm{d}}$ specifies that the cross-linked block pair can be completely noninteracting; and if so, it will only contribute to the noninteracting part of the partition function (see below).

If $i>N_{\mathrm{C}}$, we find

$$
\mathrm{U}_{\mathrm{si}}=\left[\begin{array}{lll}
\mathrm{U}_{\mathrm{d}} & 0_{4} & \mathbf{0}_{4} \\
\mathbf{0}_{4} & \mathrm{U}_{\mathrm{HH}} & \mathrm{U}_{\mathrm{HC}} \\
\mathbf{0}_{4} & 0_{4} & \mathrm{U}_{\mathrm{d}}
\end{array}\right]_{i}
$$

$\mathbf{U}_{\mathrm{d} i}$ in the upper left-hand corner of $\mathbf{U}_{\mathrm{s} i}$ accounts for the statistical weight of block pair $i$ in the completely noninteracting state of the entire dimer. $\mathbf{U}_{\mathrm{HH}}$ has been defined in eq II-4d. $\mathbf{U}_{\mathrm{HC}}$ is the terminating matrix of the interacting helical stretch and is given by 


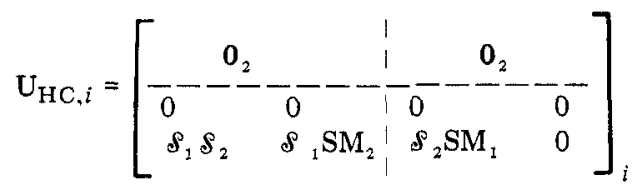

where $\mathcal{S}$ and SM are defined in eq II-1.

Finally, $\mathbf{U}_{d}$ in the lower right-hand corner of eq II- $6 \mathrm{a}$ is the statistical weight matrix of block pair $i$ that is noninteracting and lies to the right of the single interacting helical stretch. Thus, expressions for the calculation of $Z_{\mathrm{c}}{ }_{\mathrm{c}}$ have been given.

Since all internal quantities such as the helix content depend on $Z_{\mathrm{c}}^{0}$, it proves convenient to calculate $Z_{\mathrm{c}}$ directly with the $\mathbf{U}_{\mathrm{si}}$ defined in eq II-4a to II-6a, respectively. We need merely write

$$
Z_{\mathrm{c}}{ }^{0}=\mathbf{J} * \prod_{i=1}^{N_{\mathrm{B}}} \mathbf{U}_{\mathrm{si}} \mathbf{J}_{\phi}
$$

where $\mathbf{J} *$ has been defined following eq II-3b and $\mathbf{J}_{\phi}$ is a column vector containing 12 elements consisting of four ones followed by eight $r_{\phi}$ 's, wherein

$$
r_{\phi}=u_{\phi} / v_{\phi}
$$

is the ratio of the effective volume of configurational space accessible to an $\alpha$-helical block in one of the two chains in the interacting dimer when the other block is held fixed to the effective volume in configurational space of the cross-linked block, $N_{\mathrm{C}}$, in one of the two completely noninteracting chains when the other cross-linked block is held fixed. $r_{\phi}$ is a parameter to be extracted from experiment and intuitively, we would expect $r_{\phi}$ to be less than unity.

Overall Helix Content. The fraction of helix in the cross-linked dimer in the loops-excluded model is given by

$$
\begin{gathered}
f_{\mathrm{he}}{ }^{0}=f_{\mathrm{hm}} Z_{\mathrm{m}}{ }^{2} / Z_{\mathrm{c}}{ }^{0}+f_{\mathrm{d}}{ }^{0} r_{\phi} Z_{\mathrm{int}} / Z_{\mathrm{c}}{ }^{0} \\
f_{\mathrm{he}}{ }^{0}=\mathbf{J}_{\mathrm{s}} * \prod_{i=1}^{N_{\mathrm{B}}} \mathbf{A}_{\mathrm{s} i} \mathbf{J}_{\mathrm{s} \phi} /\left(Z_{\mathrm{c}}{ }^{0} \sum_{i=1}^{N_{\mathrm{B}}} m_{i}\right)
\end{gathered}
$$

in which $\mathbf{J}_{9}^{*}$ is a row vector consisting of a one followed by 23 zeros. $\mathbf{J}_{s \phi}$ is a column vector composed of 12 zeros, followed by four ones, followed by a column composed of the eight $r_{\phi} . \mathbf{A}_{\mathrm{si}}$ is a $24 \times 24$ partitioned matrix of the form

$$
\mathbf{A}_{\mathrm{s} i}=\left[\begin{array}{ll}
\mathrm{U}_{\mathrm{si}} & \mathrm{U}_{\mathrm{s} i}^{\prime} \\
\mathrm{O}_{12} & \mathrm{U}_{\mathrm{s} i}
\end{array}\right]
$$

with $\mathbf{U}_{\mathrm{s} i}$ given by eq II-4a, II-5a, or II-6a for $i<N_{\mathrm{C}}, i=$ $N_{\mathrm{C}}$, and $i>N_{\mathrm{C}}$, respectively.

Furthermore, if $i<N_{\mathrm{C}}$

$$
\mathrm{U}_{\mathrm{Si}}^{\prime}=\left[\begin{array}{lll}
\mathrm{U}_{\mathrm{d}}^{\prime} & \mathrm{U}_{\mathrm{CH}}^{\prime} & \mathbf{0}_{4} \\
\mathbf{0}_{4} & \mathrm{U}_{\mathrm{HH}}^{\prime} & \mathbf{0}_{4} \\
\mathbf{0}_{4} & \mathbf{0}_{4} & \mathbf{0}_{4}
\end{array}\right]_{i}
$$

and

$$
\mathrm{U}_{\mathrm{d}}^{\prime}=\left[\begin{array}{ll}
0 & \tau^{\prime} \\
\rho^{\prime} & m_{i} \mathrm{SM}_{i}
\end{array}\right] \otimes \mathrm{U}_{\mathrm{m} i}
$$

wherein

$$
\tau_{i}^{\prime}=\sum_{j=1}^{m_{i}}\left(m_{i}-j+1\right) \sigma_{j} \prod_{k=j}^{m_{i}} s_{k}
$$

and

$$
\mathfrak{S}_{i}^{\prime}=\sum_{j=1}^{m_{i}-1} j \prod_{k=1}^{j} s_{k}
$$

Now

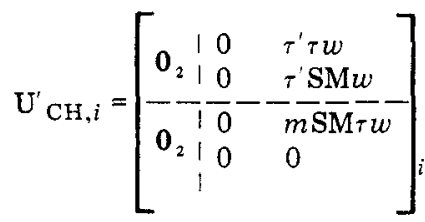

and

$$
\mathrm{U}_{\mathrm{HH}, i}^{\prime}=\left[\begin{array}{c:cc}
\mathbf{0}_{2} & 1 & \\
\hdashline 0_{2} & 0 & 0_{2} \\
\hdashline & 0 & m \mathrm{SM}^{2} w
\end{array}\right]_{i}
$$

If $i=N_{\mathrm{C}}$

$$
\mathbf{U}^{\prime}{ }_{S N_{\mathrm{C}}}=\left[\begin{array}{lll}
\mathrm{U}_{\mathrm{d}}^{\prime} & \mathrm{U}_{\mathrm{CH}, N_{\mathrm{C}}}^{\prime} & 0_{4} \\
\mathbf{0}_{4} & \mathrm{U}_{\mathrm{HH}}^{\prime} & 0_{4} \\
\mathbf{0}_{4} & \mathbf{0}_{4} & \mathbf{0}_{4}
\end{array}\right]
$$

in which $\mathbf{U}_{d}^{\prime}$ is given by eq II- $10 \mathrm{~b}$; $\mathbf{U}_{\mathrm{CH}}^{\prime}$ is given by

$$
\mathrm{U}_{\mathrm{CH}, N_{\mathrm{C}}}^{\prime}=\left[\begin{array}{l:ll}
0_{2} & 0 & \tau^{\prime} N_{\mathrm{C}} \tau_{N_{\mathrm{C}}} w \\
\hdashline 0_{2} & 0 & \tau^{\prime}{ }_{\mathrm{C}} \mathrm{SM}_{N_{\mathrm{C}}} w \\
\hdashline m_{N_{\mathrm{C}}} \mathrm{SM}_{N_{\mathrm{C}} \tau_{N_{\mathrm{C}}} w}
\end{array}\right]
$$

where $\tau_{N_{\mathrm{C}}}$ is given in eq II-5b, $m_{N_{\mathrm{C}}}$ is the total number of residues in block $N_{\mathrm{C}}$, and

$$
\tau^{\prime} N_{\mathrm{C}}=\sum_{j=1}^{m_{l}} \sigma_{j}\left(m_{N_{\mathrm{C}}}-j+1\right) \prod_{k=j}^{m_{N_{\mathrm{C}}}} s_{k}
$$

$\mathbf{U}^{\prime}{ }_{H H}$ is defined in eq II-10f with $i=N_{\mathrm{C}}$.

In the case where $i>N_{\mathrm{C}}$

$$
\mathrm{U}_{\mathbf{S i}}^{\prime}=\left[\begin{array}{lll}
\mathrm{U}^{\prime} & 0_{4} & 0_{4} \\
0_{4} & \mathrm{U}^{\prime} & \mathrm{U}^{\prime} \mathrm{HC} \\
0_{4} & \mathbf{0}_{4} & \mathrm{U}_{\mathrm{d}}^{\prime}
\end{array}\right]_{i}
$$

$\mathbf{U}_{H H}^{\prime}$ and $\mathbf{U}_{\mathrm{d}}$ are defined in eq II-10f and II-10b, respectively, and

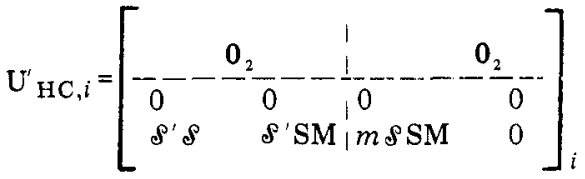

This completes the derivation of $f_{\text {he }}{ }^{0}$, the overall helix content of a cross-linked, two-chain, coiled coil treated in the context of the loops-excluded model.

Helix Probability Profiles. The helix content of the $j$ th block, $f_{h e}{ }^{0}(j)$, trivially follows from eq II-8a and is given by

$$
f_{\mathrm{he}}{ }^{0}(j)=\mathbf{J} * \prod_{i=1}^{j-1} \mathbf{U}_{\mathrm{si}} \mathbf{U}_{\mathrm{sj}}^{\prime} \prod_{i=j+1}^{N_{\mathrm{B}}} \mathbf{U}_{\mathrm{si}} \mathbf{J}_{\phi} /\left(m_{j} Z_{\mathrm{c}}{ }^{0}\right)
$$

with $\mathbf{U}_{s i}$ and $\mathbf{U}_{s i}^{\prime}$ appropriate to $i$ less than, equal to, or greater than $N_{\mathrm{C}}$ given in eq II-4a, II-5a, and II-6a and in eq II-10a, II-11a, and II-12a, respectively.

Fraction of Random Coils in Terminal Sequences. The fraction of blocks that are both randomly coiled and that are in end random coil sequences is given by

$$
f_{\mathrm{ce}}{ }^{0}=f_{\mathrm{mce}} \frac{Z_{\mathrm{m}}{ }^{2}}{Z_{\mathrm{c}}{ }^{0}}+\frac{r_{\phi}}{N_{\mathrm{B}} Z_{\mathrm{c}}{ }^{0}}\left\{\mathbf{J}_{\mathrm{s}} * \prod_{i=1}^{N_{\mathrm{B}}} \mathbf{A}_{\mathrm{fs} i} \mathbf{J}_{\mathrm{s}}+\mathbf{J}_{\mathrm{s}} * \prod_{i=1}^{N_{\mathrm{B}}} A_{\mathrm{bs} i} \mathbf{J}_{\mathrm{s}}\right\}
$$

$f_{\text {mce }}$ is the fraction of randomly coiled blocks in terminal random coil sequences in an isolated single chain; a prescription for the calculation of $f_{\text {mce }}$ is given below. $\mathbf{J}_{\mathrm{s}}$ is 
a column vector consisting of 16 zeros follows by eight ones. Now $A_{\mathrm{fa} i}$ is a $24 \times 24$ partitioned matrix

$$
\mathbf{A}_{\mathrm{fs} i}=\left[\begin{array}{ll}
\mathrm{U}_{\mathrm{fs}} & \mathrm{U}_{\mathrm{fs}} \\
0_{12} & \mathrm{U}_{\mathrm{s}}
\end{array}\right]_{i}
$$

in which $\mathrm{U}_{\mathrm{s} i}$ is given by eq II-4a, II-5a, or II-6a for $i$ less than, equal to, or greater than $N_{\mathrm{C}}$, respectively. If $i \leq N_{\mathrm{C}}$

$$
\mathrm{U}_{\mathrm{fsi}}=\left[\begin{array}{lll}
\mathrm{U}_{\mathrm{fd}} & \mathrm{U}_{\mathrm{fCH}} & 0_{4} \\
\mathbf{0}_{4} & 0_{4} & 0_{4} \\
\mathbf{0}_{4} & \mathbf{0}_{4} & \mathbf{0}_{4}
\end{array}\right] i
$$

in which

$$
\mathrm{U}_{\mathrm{fd} i}=\left[\begin{array}{cc}
1 & \tau_{\mathrm{p}} \\
0 & 0
\end{array}\right]_{i} \otimes \mathrm{U}_{\mathrm{m} i}
$$

where

$$
\tau_{\mathbf{p}}=\sum_{j=1}^{m_{i}}\left[(j-1) / m_{i}\right] \sigma_{j} \prod_{k=j}^{m_{j}} s_{k}
$$

and

$$
\mathrm{U}_{\mathrm{fCH}, i}=\left[\begin{array}{c:lc}
\mathbf{0}_{2} & 10 & \tau_{\mathrm{p}} \tau w \\
\hdashline \mathbf{0}_{2} & \mathbf{0}_{2} & \tau_{\mathbf{p}} \mathrm{SMw} \\
\hdashline
\end{array}\right]_{i}
$$

wherein $\tau$ is given by eq II-1 if $i \neq N_{\mathrm{C}}$ and eq II-5b if $i=$ $N_{\mathrm{C}}$. Furthermore

$$
\tau_{\mathrm{p}, N_{\mathrm{C}}}=\sum_{j=1}^{m_{l}}\left[(j-1) / m_{N_{\mathrm{C}}}\right] \prod_{k=j}^{m_{N_{\mathrm{C}}}} s_{k}
$$

Moreover, if $i>N_{\mathrm{C}}$

$$
\mathrm{U}_{\mathrm{fs} i}=\left[\begin{array}{lll}
\mathrm{U}_{\mathrm{fd}} & \mathbf{0}_{4} & \mathbf{0}_{4} \\
\mathbf{0}_{4} & \mathbf{0}_{4} & \mathbf{0}_{4} \\
\mathbf{0}_{4} & \mathbf{0}_{4} & \mathbf{0}_{4}
\end{array}\right]_{i}
$$

Equation II-15g specifies that in the loops-excluded model of a cross-linked, two-chain, coiled coil for chains containing at least one pair of interacting blocks, block $N_{\mathrm{C}}$ must be in an interacting helical state. Thus there are no random coil sequences unwinding from the left with $i>$ $N_{\mathrm{C}}$ unless the molecule is in a noninteracting conformation.

$\mathbf{A}_{\mathrm{bsi}}$ of eq II-14 is defined as

$$
\mathbf{A}_{\mathrm{bs} i}=\left[\begin{array}{ll}
\mathrm{U}_{\mathrm{si}} & \mathrm{U}_{\mathrm{bs} i} \\
\mathbf{0}_{12} & \mathrm{U}_{\mathrm{bs} i}
\end{array}\right]
$$

in which $\mathbf{U}_{\mathbf{s i}}$ is given in eq II-4a, eq II-5a, or II-6a for $i<$ $N_{\mathrm{C}}, i=N_{\mathrm{C}}$, and $i>N_{\mathrm{C}}$, respectively.

If $i \leq N_{\mathrm{C}}$

$$
\mathbf{U}_{\mathrm{bsi}}=\mathbf{0}_{12}
$$

There are no interior randomly coiled blocks that unwind from the $\mathrm{C}$ terminus past the cross-link in those chains having a pair of interacting helices.

If $i>N_{\mathrm{C}}$, we have

$$
\begin{aligned}
& \mathrm{U}_{\mathrm{bs} i}=\left[\begin{array}{lll}
\mathbf{0}_{4} & \mathbf{0}_{4} & \mathbf{0}_{4} \\
\mathbf{0}_{4} & \mathbf{0}_{4} & \mathrm{U}_{\mathrm{bHC}} \\
\mathbf{0}_{4} & \mathbf{0}_{4} & \mathrm{U}_{\mathrm{bd}}
\end{array}\right]_{i} \\
& \mathrm{U}_{\mathrm{bHC}, i}=\left[\begin{array}{ll|l} 
& \mathbf{0}_{2} & \mathbf{0}_{2} \\
\hdashline 0 & 0 & \mathbf{0}_{2} \\
\rho_{p} \rho & \delta_{p} \mathrm{SM}
\end{array}\right] i
\end{aligned}
$$

where

$$
\rho_{p}=1+\sum_{j=1}^{m_{i}-1} \frac{m_{i}-j}{m_{i}} \prod_{k=1}^{j} s_{k}
$$

and

$$
\mathrm{U}_{\mathrm{bd} i}=\left[\begin{array}{ll}
1 & 0 \\
\mathfrak{\rho}_{p} & 0
\end{array}\right]_{i} \otimes \mathrm{U}_{m i}
$$

The fraction of end random coils in the completely noninteracting state of the dimer may be calculated from $f_{\text {mce }}=$

$$
\left\{\mathbf{J}_{\mathrm{g}} * \prod_{i=1}^{N_{\mathrm{B}}} \mathbf{A}_{\mathrm{fs} i} \mathbf{J}_{\mathrm{sm}}+\mathbf{J}_{\mathrm{bm}} * \prod_{i=1}^{N_{\mathrm{B}}} \mathbf{A}_{\mathrm{bm} i} \mathbf{J}_{\mathrm{bm}}-N_{\mathrm{B}} Z_{\mathrm{m}}\right\} /\left(N_{\mathrm{B}} Z_{\mathrm{m}}{ }^{2}\right)
$$

where $\mathbf{J}_{\mathrm{sm}}$ is a column vector of 12 zeros, followed by four ones, followed by eight zeros. $\mathbf{J}_{\mathrm{bm}}$ * is a row vector containing eight elements, the first of which is unity and the rest are zero, and $\mathbf{J}_{\mathrm{bm}}$ is a column vector of eight elements, the first four of which are zero and the last four of which are unity. Moreover

$$
\mathrm{A}_{\mathrm{bmi} i}=\left[\begin{array}{cc}
\mathrm{U}_{\mathrm{d}} & \mathrm{U}_{\mathrm{bd}} \\
0_{4} & \mathrm{U}_{\mathrm{bd}}
\end{array}\right]
$$

This completes the development of the theory of the loops-excluded model of the helix-coil transition in twochain, coiled coils.

C. Interior Eyelet Model of Singly Cross-Linked, Two-Chain, Coiled Coils. In this section, we develop the theory of the helix-to-random coil transition in a singly cross-linked, $\alpha$-helical, two-chain, coiled coil that may contain constrained interior random coil loops that originate at the cross-link and terminate on an interacting helical stretch. There may be an eyelet to the left and/or to the right of the pair of cross-linked residues located in block $N_{\mathrm{C}}$. Due to steric constraints, as mentioned in section I, we assume that the residues in the cross-linked block are all randomly coiled if they are in an interior random coil loop preceded or followed by an interacting helical stretch. Furthermore, we shall consider two kinds of interior random coil loops. The first kind, type I, consists of essentially completely (with the exception of interfacial $[\mathrm{C}] \mathrm{H}$ and $[\mathrm{H}] \mathrm{C}$ states discussed below) random coil states in both chains. The second kind, type II, consists of a completely helical sequence propagating from block $i$ to the cross-link in one of the two chains and a completely random coil sequence, containing $k$ residues, propagating from block $i$ to the cross-link in the other chain. Hence in a type II loop, the $k$ random coiled residues are contrained to lie, within a certain tolerance, at a distance $|\mathbf{r}|=k p / m$, wherein $p$ is the pitch of the $\alpha$-helix (approximately $5.4 \AA /$ turn $^{32}$ ) and $m$ is the number of residues per turn. Thus, $p / m$ is the axial translation per residue and will be taken to be $1.5 \AA$ /residue in what follows.

Let the $i$ th-block pair be completely helical. Define $k_{1}$ and $k_{2}$ as the net number of random coiled residues in the loop in chains one and two, respectively, and let $k_{\mathrm{H}}$ be the net difference in helical states in the two chains propagating from block pair $i+1(i-1)$ to the cross-link if $i<$ $N_{\mathrm{C}}$ (if $i>N_{\mathrm{C}}$ ). The statistical weight of the $k_{1}$ and $k_{2}$ random coil residues constrained to lie at a distance $k_{\mathrm{H}^{-}}$ $(p / m)$ is for a Gaussian chain equal to

$$
\begin{aligned}
& u_{\phi} \delta\left(k_{1}+k_{2} ; k_{\mathrm{H}}\right)= \\
& \frac{C_{1}}{\left(k_{1}+k_{2}+l_{c}\right)^{3 / 2}} \exp \left\{-\frac{3}{2} k_{\mathrm{H}}^{2}(p / m)^{2} /\left(k_{1}+k_{2}+l_{c}\right) b_{0}^{2}\right\}
\end{aligned}
$$

in which 


$$
C_{1}=(3 / 2 \pi)^{3 / 2} u_{\phi} b_{0}{ }^{-3}
$$

wherein $b_{0}$ is the effective bond length per residue and is taken to be $8.0 \AA$ in what follows. ${ }^{33}$ The quantity $C_{1} /\left(k_{1}\right.$ $\left.k_{2}+l_{c}\right)^{3 / 2}$ physically represents the ratio of the volume of configurational space accessible to a random coil composed of $k_{1}+k_{2}+l_{c}$ residues when the ends are constrained to lie with an effective volume $u_{\phi}$ relative to the case when the ends are free. If $b_{u}$ is the virtual bond length between $\mathrm{C}^{\alpha}$ carbon centers in the polypeptide backbone (taken equal to $3.80 \AA^{31}$ ), then we have assumed in writing eq II-20a that $l_{c} b_{\mathrm{u}}$ is the distance between $\mathrm{C}^{\alpha}$ carbons of the cross-linked residues in the two chains and that the cross-link behaves as part of a Gaussian chain having the same effective bond length as the polypeptide backbone. This assumption can be easily removed if $b_{0}$ of the cross-link is known. Moreover, while we shall employ eq II-20a to account for the effects of loop entropy, the formalism developed below does not require it; in principle, any function of $k_{1}, k_{2}$, and $k_{\mathrm{H}}$ can be employed.

There is one additional point that needs to be addressed. Each interacting pair of helices contains a factor in the partition function to account for the available phase space when the first pair of $\alpha$-helical turns in the two chains are brought into contact and aligned. If there are no interior random coil loops between interacting helices attached to and including the cross-linked block, the factor $u_{\phi}$ alone is required. If there are such closed loops, the factor $u_{\phi} \delta\left(k_{1}\right.$ $+k_{2} ; k_{\mathrm{H}}$ ) is called for. Hence, there is always a factor of $u_{\phi}$ for each pair of $\mathbf{U}_{\mathrm{CH}}$ and $\mathbf{U}_{\mathrm{HC}}$ (initiating and terminating states of the interacting helical stretch) matrices of the interacting helical stretch. Since there must be at least one $u_{\phi}$ per interacting dimer, we append the first $u_{\phi}$ factor (actually $r_{\phi}$ defined in eq II-7b) to the column vector postmultiplying the supermatrix expressions developed below.

Construction of the Partition Function. The partition function of a singly cross-linked, two-chain, coiled coil in the interior eyelet model is given by

$$
Z_{\mathrm{d}}=v_{\phi} Z_{\mathrm{c}}
$$

wherein

$$
\begin{gathered}
Z_{\mathrm{c}}=Z_{\mathrm{m}}{ }^{2}+r_{\phi} Z_{\mathrm{int}} \\
Z_{\mathrm{c}}=Z_{\mathrm{m}}{ }^{2}+\mathrm{J} * \prod_{i=1}^{N_{\mathrm{B}}} \mathbf{U}_{\mathrm{se} i} \mathbf{J}_{\phi}
\end{gathered}
$$

where $Z_{\mathrm{m}}{ }^{2}$ is the partition function associated with the noninteracting conformation of the singly cross-linked chains, $\mathbf{J}^{*}$ has been defined following eq II- $3 \mathrm{~b}$, and $\mathbf{J}_{\phi}$ has been defined followed eq II-7a.

Three cases of $\mathbf{U}_{\text {sei }}$, need to be explicitly examined. We begin with the case $i<N_{\mathrm{C}}$.

$$
\mathrm{U}_{\mathrm{se} i}=\left[\begin{array}{lll}
\mathrm{U}_{\mathrm{d}} & \mathrm{U}_{\mathrm{CH}} & \mathbf{0}_{4} \\
\mathbf{0}_{4} & \mathrm{U}_{\mathrm{HH}} & \mathbf{U}^{l} \mathrm{HC} \\
\mathbf{0}_{4} & \mathbf{0}_{4} & \mathbf{E}_{4}
\end{array}\right]_{i}
$$

$\mathbf{U}_{\mathrm{d}}, \mathbf{U}_{\mathrm{CH}}$, and $\mathbf{U}_{\mathrm{HH}}$ are defined in eq II-4b to II- $4 \mathrm{~d}$, respectively. $\mathbf{U}^{l}{ }_{\mathrm{HC}}$ explicitly accounts for the type I and II interior random coil loops propagating from block $i$ to the cross-link $N_{\mathrm{C}}$.

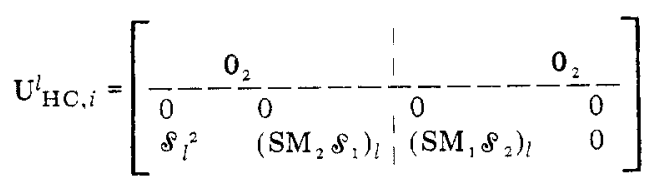

in which 1 and 2 refer to chains one and two, respectively, and

$$
\begin{aligned}
& \mathcal{S}_{l}^{2}=\delta\left(2 \sum_{j=1}^{N_{\mathrm{C}^{-1}}} m_{j}+2 m_{l}+l_{c} ; 0\right)+ \\
& 2 \sum_{r=1}^{m_{i}-1} \prod_{k=1}^{r} s_{k} \delta\left(2 \sum_{j=i+1}^{N_{C^{-1}}} m_{j}+2 m_{l}+l_{c}+2 m_{i}-r ; r\right)+\sum_{n=1}^{m_{-}-1} \sum_{r=1}^{m_{l}-1} \\
& \quad \prod_{k=1}^{n} s_{k} \prod_{l=1}^{r} s_{l} \delta\left(2 \sum_{j=i+1}^{N_{\mathrm{C}}-1} m_{j}+2 m_{l}+l_{c}+2 m_{i}-n-r ;|n-r|\right)
\end{aligned}
$$

accounts for the loop entropy of a type I random coil loop.

$$
\begin{aligned}
& \left(\mathrm{SM}_{\alpha} \mathfrak{S}_{\beta}\right)_{l}= \\
& \prod_{j=i}^{N_{\mathrm{C}}-1} \mathrm{SM}_{j, \alpha}\left[\delta\left(\sum_{k=i}^{N_{\mathrm{C}^{-1}}} m_{k}+m_{l}+l_{c} ; \sum_{k=i}^{N_{\mathrm{C}^{-1}}} m_{k}+m_{l}\right)+\right. \\
& \left.\sum_{r=1}^{m_{l}-1} \prod_{k=1}^{r} s_{k} \delta\left(\sum_{n=i+1}^{N_{\mathrm{C}^{-1}}} m_{n}+m_{l}+l_{\mathrm{c}}+m_{i}-r ; \sum_{n=i}^{N_{\mathrm{C}^{-1}}} m_{n}+m_{l}-r\right)\right]
\end{aligned}
$$

The indices $\alpha$ and $\beta$ refer to chains $\alpha$ and $\beta$, respectively. Equation II-22d accounts for the loop entropy of a type II constrained interior random coil loop.

The $4 \times 4$ identity matrix, $\mathbf{E}_{4}$ in the lower right-hand corner of eq II-22a specifies that the statistical weights of block pair $i$ in all interior closed loops originating at blocks $j<i$ and including block $i$ are already accounted for in $\mathbf{U}_{\mathrm{HC}, j}^{l}, j<i$.

It is worthwhile to compare eq II-22a with eq II-4a; in the latter equation, $\mathbf{E}_{4}$ and $\mathbf{U}^{l}$ HC have been set equal to zero, while in the former equation, the statistical weights of type I and II randomly coil loops are explicitly accounted for.

If $i=N_{\mathrm{C}}$, we have

$$
\mathrm{U}_{\mathrm{se} N_{\mathrm{C}}}=\left[\begin{array}{lll}
\mathrm{E}_{4} & \mathrm{U}_{\mathrm{CH}, N_{\mathrm{C}}} & \mathbf{0}_{4} \\
u_{\phi} \mathrm{U}_{\mathrm{HC}}^{l} & \mathrm{U}_{\mathrm{HH}} & \mathrm{U}_{\mathrm{HC}}^{l} \mathrm{U}_{N_{\mathrm{C}}} \\
u_{\phi} \mathbf{E}_{4} & 0_{4} & \mathrm{U}_{N_{\mathrm{C}}}
\end{array}\right]
$$

$\mathbf{E}_{4}$ in the upper left-hand corner accounts for the possibility that the cross-linked block is randomly coiled and contains at least one interacting helical stretch to the righ and has no interacting helical stretches to the left. All such interior random coil loops to the right will be accounted for by the $\mathbf{U}_{\mathrm{CH}}$ matrices (see eq II-26a below). The $\mathbf{U}_{\mathrm{HC}}^{l}$ and $\mathbf{E}_{4}$ matrices in the lower left of eq II-23a allow for the "double-eyelet" states, namely a $\mathbf{U}_{\mathrm{HC}}^{l} \mathbf{U}_{\mathrm{CH}}^{r}$ type state. The factor $u_{\phi}$ as mentioned above is necessary to correctly account for the presence of the second pair of interacting helices. $\mathrm{U}_{\mathrm{CH}, N_{\mathrm{C}}}$ is given by eq II-4c but with $\tau_{N_{\mathrm{C}}}$ defined in eq II-5b substituted for $\tau$. $\mathbf{U}^{l}{ }_{\mathrm{HC}, N_{\mathrm{C}}}$ is of the same form as eq II-22b but in which

$$
\begin{gathered}
\mathcal{S}_{l}^{2}=\delta\left(2 m_{l}+l_{c} ; 0\right) \\
\left(\mathrm{SM}_{\alpha} \mathcal{S}_{\beta}\right)_{l}=\delta\left(m_{l}+l_{c} ; m_{l}\right)
\end{gathered}
$$

Equations II-23b and II-23c specify that in the $\mathbf{U}_{\mathrm{HC}}^{l}$-type state located at $N_{\mathrm{C}}$, the residues to the left of the crosslinked residue are in completely randomly coiled type I and II loops. The residues to the right of the cross-link are also, as indicated previously, assumed to be randomly coiled. The $\mathbf{U}_{\mathrm{HC}, N_{\mathrm{C}}}^{l}$ matrix in the far right columns of $\mathbf{U}_{\mathrm{se} N_{\mathrm{C}}}$ couples the randomly coiled cross-linked block having the smallest size interior random coil loop to the noninteracting states at the right of the cross-link.

Finally

$$
\mathrm{U}_{N_{\mathrm{C}}}=\left[\begin{array}{llll}
1 & 0 & 0 & 0 \\
0 & \mathrm{SM}_{N_{\mathrm{C}}} & 0 & 0 \\
0 & 0 & \mathrm{SM}_{N_{\mathrm{C}}} & 0 \\
0 & 0 & 0 & 0
\end{array}\right]
$$


and couples those states having a pair of interacting helices and constrained interior random coiled loops involving blocks $j<N_{\mathrm{C}}$ to the noninteracting states in the half of the molecule with $j>N_{\mathrm{C}} . \mathrm{SM}_{N_{\mathrm{C}}}$ appears in eq II-24 rather than in eq II-22d to avoid overcounting the fully helical cross-linked block when $\mathrm{U}_{\mathrm{CH}^{-}}$-type states are included (see eq II-26a below).

Equation II-23a should be contrasted with eq II-5a, where the possibility of type I or type II states is excluded.

When $i>N_{\mathrm{C}}$, we have a matrix isomorphic with the broken $\alpha$-helical hairpin model (see eq II-5b of ref 34 ) for single chains having interhelical contacts developed previously.

$$
\mathrm{U}_{\mathrm{sei}}=\left[\begin{array}{lll}
\mathrm{E}_{4} & \mathrm{U}_{\mathrm{CH}}^{r} & 0_{4} \\
0_{4} & \mathrm{U}_{\mathrm{HH}} & \mathrm{U}_{\mathrm{HC}} \\
0_{4} & 0_{4} & \mathrm{U}_{\mathrm{d}}
\end{array}\right]_{i}
$$

with

$$
\begin{aligned}
& \mathrm{U}_{\mathrm{CH}, i}^{r}=\left[\begin{array}{c:cc}
\mathbf{0}_{2} & 0 & \left(\tau^{2}\right)_{l} w \\
\hdashline \mathbf{0}_{2} & 0 & 0 \\
\hdashline & 0 & \left.\left(\tau_{2} \mathbf{S M}_{2}\right)_{l} \mathbf{S M}_{1}\right)_{l} w
\end{array}\right]_{i} \\
& \tau_{l}^{2}=\sum_{j=1}^{m_{i}} \sum_{r=1}^{m_{i}} \sigma_{j} \sigma_{r} \prod_{k=j}^{m_{i}} s_{k} s_{l} \delta\left(2 \sum_{n=N_{\mathrm{C}}+1}^{i-1} m_{n}+2 m_{r}+l_{c}+j+\right. \\
& r-2 ;|j-r|)
\end{aligned}
$$

and

$$
\begin{aligned}
\left(\tau_{\alpha} \mathbf{S M}_{\beta}\right)_{l}= & \prod_{j=N_{\mathrm{C}}}^{i} \mathbf{S M}_{j, \beta}{ }^{\alpha} \sum_{r=1}^{m_{i}} \sigma_{r} \prod_{k=r}^{m_{i}} s_{k} \delta\left(\sum_{n=N_{\mathrm{C}}+1}^{i-1} m_{n}+m_{r}+l_{\mathrm{c}}+\right. \\
& \left.r-1 ; \sum_{n=N_{\mathrm{C}}+1}^{i} m_{n}+m_{r}-\left(m_{i}-r+1\right)\right)(\mathrm{II}-26
\end{aligned}
$$

That is, $\mathbf{U}^{r} \mathrm{CH}$ accounts for the statistical weight of an interacting helical stretch starting at block $i>N_{\mathrm{C}}$ and containing a type I or type II loop propagating from the cross-linked block. Observe that in contrast to the broken $\alpha$-helical model developed previously for a single-chain polypeptide having interhelical contacts, ${ }^{34}$ we include type II as well as type I loops.

$\mathbf{E}_{4}$ in eq II-25 takes account of type I and type II interior random coil sequences including block $i$ and having an interacting pair of helices that initiates at block $j>i$. $\mathbf{U}_{\mathrm{HH}}$ has been defined in eq II-4a, and $\mathbf{U}_{\mathrm{HC}}$ is given by eq II- $6 \mathrm{~b}$.

The reader is urged to compare eq II-25 with eq II-6a, where the possibility of interior random coil states propagating from the cross-link is prohibited.

It should be pointed out that the supermatrix experssion for $Z_{c}$, eq II-21c, generates all type I and type II eyelets centered about the pair of cross-linked blocks as well as all conformations containing a single interacting pair of $\alpha$-helices that propagate through the cross-link.

Overall Helix Content. The helix content of a cross-linked, two-chain, coiled coil, $f_{\text {he, }}$, is given in the interior eyelet model by

$$
\begin{gathered}
f_{\mathrm{he}}=f_{\mathrm{hm}} Z_{\mathrm{m}}^{2} / Z_{\mathrm{c}}+f_{\mathrm{d}} r_{\phi} Z_{\text {int }} / Z_{\mathrm{c}} \\
f_{\mathrm{he}}=f_{\mathrm{hm}} Z_{\mathrm{m}}^{2} / Z_{\mathrm{c}}+\mathbf{J}_{\mathrm{s}} * \prod_{i=1}^{N_{\mathrm{B}}} \mathbf{A}_{\mathrm{se} i} \mathbf{J}_{\mathrm{se}} / N_{\mathrm{T}} Z_{\mathrm{c}}
\end{gathered}
$$

Here, $f_{\mathrm{hm}}$ is the helix content of a chain lacking any interhelical contacts whatsoever (assumed equivalent to that in an isolated single chain; see eq 28 of ref 1 ). $\mathbf{J}_{\mathrm{s}}{ }^{*}$ has been already defined immediately following eq II-8b, $\mathbf{J}_{\mathrm{se}}$ is a column vector containing 24 elements composed of 16 zeros followed by eight " $r_{\phi}$ ", and $N_{\mathrm{T}}$ is the total number of residues per chain. Since the two chains are assumed to be identical, though not necessarily homopolymeric, we need only focus on the helix content of chain one.

$\mathbf{A}_{\text {se } i}$ is a $24 \times 24$ partitioned supermatrix having the structure

$$
\mathbf{A}_{\text {se } i}=\left[\begin{array}{ll}
\mathrm{U}_{\mathrm{se} i} & \mathrm{U}_{\mathrm{se} i}^{\prime} \\
\mathbf{0}_{12} & \mathrm{U}_{\mathrm{se} i}
\end{array}\right]
$$

where $\mathrm{U}_{\text {sei }}$ has been defined in eq II-22a, II-23a, or II-25 for $i$ less than, equal to, or greater than $N_{\mathrm{C}}$, respectively. Since we are interested here in the overall helix content rather than the helix probability profile, formally $\mathbf{U}_{\text {se } i}^{\prime}$ is defined as

$$
\sum_{j=1}^{N_{\mathrm{B}}} \sum_{k=1}^{m_{j}} \frac{\partial \mathbf{U}_{\mathrm{se} i}}{\partial \ln s_{k j}}
$$

where in $s_{k j}$ is the Zimm-Bragg propagation parameter associated with the $k$ th residue in the $j$ th block in chain one. Previously, the above expression contained nonzero terms only for $i=j ;^{1-4}$ however, due to the inclusion of completely helical states that propagate from block $i$ to block $N_{\mathrm{C}}$ in a type II loop (eq II-22d and II-26c) this is not the case here.

In particular, on setting $i<N_{\mathrm{C}}$

$$
\mathrm{U}_{\text {se } i}^{\prime}=\left[\begin{array}{lll}
\mathrm{U}_{\mathrm{d}}^{\prime} & \mathrm{U}_{\mathrm{CH}}^{\prime} & \mathbf{0} \\
\mathbf{O}_{4} & \mathrm{U}_{\mathrm{HH}}^{\prime} & \mathrm{U}^{\prime \prime} \mathrm{HC} \\
\mathbf{0}_{4} & \mathbf{0}_{4} & \mathbf{0}_{4}
\end{array}\right]_{i}
$$

$\mathbf{U}_{d}^{\prime}, \mathbf{U}^{\prime}{ }_{\mathrm{CH}}$, and $\mathbf{U}_{\mathrm{HH}}^{\prime}$ are defined in eq II-10b, II-10e, and II-10f, respectively, and

$$
\mathrm{U}^{\prime}{ }_{\mathrm{HC}, i}=\left[\begin{array}{llll} 
& \mathbf{0}_{2} & \mathbf{0} & \mathbf{0}_{\mathbf{2}} \\
\hdashline 0 & 0 & 0 & 0 \\
\left(\mathcal{f} \mathcal{S}^{\prime}\right)_{l} & \left(\mathrm{SM}^{\prime}\right)_{l} & \left(\mathrm{SM}^{\prime} \mathcal{S}\right)_{l} & 0
\end{array}\right]_{i}
$$

We have dropped the subscripts 1 and 2 for convenience (a prime always refers to chain one), and it immediately follows from eq II-22c,d that

$$
\begin{aligned}
& \left(\mathcal{S}^{\prime}\right)_{l}=\sum_{r=1}^{m_{l}-1} r \prod_{k=1}^{r} s_{k} \delta\left(2 \sum_{j=i+1}^{N_{\mathrm{C}^{-1}}} m_{j}+2 m_{l}+l_{\mathrm{c}}+2 m_{i}-r ; r\right)+ \\
& \sum_{n=1}^{m_{i}-1} \sum_{r=1}^{m_{l}-1} r \prod_{k=1}^{n} s_{k} \prod_{l=1}^{r} s_{l} \delta\left(2 \sum_{j=i+1}^{N_{\mathrm{C}}-1} m_{j}+2 m_{l}+l_{\mathbf{c}}+\right. \\
& \left.2 m_{i}-r-n ;|n-r|\right) \\
& \left(\mathrm{SM}^{\prime}\right)_{l}=\prod_{j=i}^{N_{\mathrm{C}^{-1}}} \mathrm{SM}_{j}\left[\sum _ { r = 1 } ^ { m _ { i } - 1 } r \prod _ { k = 1 } ^ { r } s _ { k } \delta \left(\sum_{n=i+1}^{N_{\mathrm{C}}-1} m_{n}+m_{l}+l_{\mathrm{c}}+m_{i}-\right.\right. \\
& \left.\left.r ; \sum_{j=i}^{N_{C^{-1}}} m_{n}+m_{l}-r\right)\right]
\end{aligned}
$$

and

$$
\left(\mathrm{SM}^{\prime} \mathfrak{S}\right)_{l}=\sum_{j=i}^{N_{\mathrm{C}^{-1}}} m_{j}(\mathrm{SM} \mathcal{S})_{l}
$$

$(\mathrm{SM} \mathcal{})_{l}$ is defined in eq II-22d. In eq II-29e, the nondiagonal nature of $\mathbf{U}^{\prime}{ }_{8 i}$ (i.e., the inclusion of $i \neq j$ terms in eq II-28b) becomes immediately apparent.

If we let $i=N_{\mathrm{C}}$, then

$$
\mathrm{U}^{\prime}{ }_{\mathrm{se} N_{\mathrm{C}}}=\left[\begin{array}{lll}
\mathbf{0}_{4} & \mathrm{U}_{\mathrm{CH}, N_{\mathrm{C}}} & \mathbf{0}_{4} \\
\mathbf{0}_{4} & \mathrm{U}^{\prime}{ }_{\mathrm{HH}} & \mathrm{U}^{\prime \prime}{ }_{\mathrm{HC}_{\mathrm{C}}} \mathrm{U}_{N_{\mathrm{C}}} \\
\mathbf{0}_{4} & \mathbf{0}_{4} & \mathrm{U}^{\prime}{ }_{N_{\mathrm{C}}}
\end{array}\right]
$$

In eq II-30a, $\mathbf{U}^{\prime}{ }_{\mathrm{CH}, N_{\mathrm{C}}}$ is given in eq II- $11 \mathrm{~b}, \mathbf{U}^{\prime}{ }_{\mathrm{HH}}$ is found in eq II-10f, $\mathbf{U}_{N_{C}}$ is given in eq II-24, and 


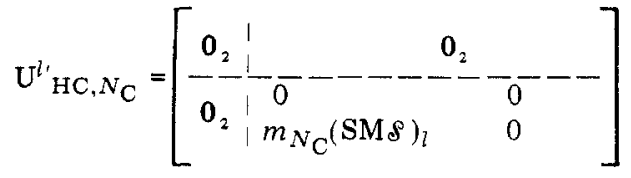

with $(\mathrm{SM} P)_{l}$ defined in eq II-23c. Furthermore

$$
\mathrm{U}_{N_{\mathrm{C}}}=\left[\begin{array}{llll}
0 & 0 & 0 & 0 \\
0 & 0 & 0 & 0 \\
0 & 0 & m_{N_{\mathrm{C}}} \mathrm{SM}_{N_{\mathrm{C}}} & 0 \\
0 & 0 & 0 & 0
\end{array}\right]
$$

with $\mathrm{SM}_{N_{\mathrm{C}}}$ defined in eq II-1.

Finally, if $i>N_{\mathrm{C}}$, we find

$$
\mathbf{U}_{\text {sei }}^{\prime}=\left[\begin{array}{lll}
\mathbf{0}_{4} & \mathrm{U}^{\prime \prime} \mathrm{CH} & \mathbf{0}_{4}^{\prime} \\
\mathbf{0}_{4} & \mathrm{U}_{\mathrm{HH}}^{\prime} & \mathbf{U}^{\prime}{ }_{\mathrm{HC}} \\
\mathbf{0}_{4} & \mathbf{0}_{4} & \mathrm{U}_{\mathrm{d}}^{\prime}
\end{array}\right]_{i}
$$

$\mathbf{U}^{\prime}{ }_{H H}, \mathbf{U}^{\prime}{ }_{H C}$, and $\mathbf{U}^{\prime}{ }_{d}$ are the standard, non-cross-linked propagating, terminating, and noninteracting $4 \times 4$ statistical weight matrices of eq II-10f, II-12b, and II-10b, respectively. Moreover

$$
\mathrm{U}^{\prime}{ }_{\mathrm{CH}, i}=\left[\begin{array}{c:ll}
\mathbf{0}_{2} & 0 & \left(\tau \tau^{\prime}\right)_{l} w \\
\hdashline \mathbf{0}_{2} & 0 & 0 \\
\hdashline & 0 & 0
\end{array}\right]_{i}^{\left(\tau \mathbf{S M}^{\prime}\right)_{l} w}
$$

(II-31b)

wherein

$$
\begin{gathered}
\left(\tau \tau^{\prime}\right)_{l}=\sum_{j=1}^{m_{i}} \sum_{r=1}^{m_{i}}\left(m_{i}-j+1\right) \sigma_{j} \sigma_{r} \prod_{\substack{k \\
l=j}}^{m_{i}} s_{k} s_{l} \delta\left(2 \sum_{n=N_{\mathrm{C}}+1}^{i-1} m_{n}+\right. \\
\left.2 m_{r}+l_{\mathrm{c}}+j+r-2 ;|j-r|\right)(\mathrm{II}-31 \mathrm{c}) \\
\left(\tau^{\prime} \mathrm{SM}\right)_{l}=\prod_{j=N_{\mathrm{C}}}^{i} \mathrm{SM}_{j} \sum_{r=1}^{m_{i}}\left(m_{i}-r+1\right) \sigma_{r} \prod_{k=r}^{m_{i}} s_{k} \delta\left(\sum_{n=N_{\mathrm{C}}+1}^{i-1} m_{n}+\right. \\
\left.m_{r}+l_{\mathrm{c}}+r-1 ; \sum_{n=N_{\mathrm{C}}+1}^{i} m_{n}+m_{r}-\left(m_{i}-r+1\right)\right)(\mathrm{II}-31 \mathrm{~d})
\end{gathered}
$$

and

$$
\left(\tau \mathrm{SM}^{\prime}\right)_{l}=\sum_{j=N_{\mathrm{C}}}^{i} m_{j}(\tau \mathrm{SM})_{l}
$$

$(\tau \mathrm{SM})_{l}$ is defined in eq II-26c. This completes the presentation of the expressions required to calculate the overall helix content of a singly cross-linked, two-chain, coiled coil, $f_{\text {he. }}$

Helix Probability Profiles. Since the $j$ th residue may be in a helical state as part of a noninteracting helical stretch not involved in the type II random coil loop attached to the cross-link, in a type II loop, or in an interacting helical stretch, we shall require the product of $24 \times 24$ supermatrices to obtain the helix content of the $j$ th block.

$$
f_{\mathrm{he}}(j)=f_{\mathrm{hm}}(j) \frac{Z_{\mathrm{m}}^{2}}{Z_{\mathrm{c}}}+\mathbf{J}_{\mathrm{s}} * \prod_{i=1}^{N_{\mathrm{p}}} \mathbf{A}_{\mathrm{spi}}^{j} \mathbf{J}_{\mathrm{se}} /\left(m_{\mathrm{j}} Z_{\mathrm{c}}\right)
$$

in which $f_{\mathrm{hm}}(j)$ is the helix content of the $j$ th residue in an isolated single chain and may be obtained from eq II-14 of ref $2 . \mathbf{A}_{\mathrm{sp} i}^{j}$ is of the form

$$
\mathbf{A}_{\text {spi }}^{j}=\left[\begin{array}{ll}
\mathbf{U}_{\text {sei }} & \mathbf{U}^{j \cdot} \\
\mathbf{0}_{12} & \mathbf{U}_{\mathrm{sei} i}
\end{array}\right]
$$

in which $\mathrm{U}_{\mathrm{se}}$ is given in eq II-22a, II-23a, and II-25 for $i$ less than, equal to, or greater than $N_{\mathrm{C}}$ and

$$
\mathbf{U}^{j \prime}{ }_{s e i}=\sum_{k=1}^{m_{j}} \frac{\partial \mathbf{U}_{s e i}}{\partial \ln s_{j k}}
$$

In what follows we need merely evaluate eq II- $33 \mathrm{~b}$ for the three different cases as a function of $i$ where $j<N_{\mathrm{C}}, j=$ $N_{\mathrm{C}}$, and $j>N_{\mathrm{C}}$.

Case 1: $\boldsymbol{j}<\boldsymbol{N}_{\mathrm{C}}$. If $j<i$, it immediately follows using eq II-22a in eq II-33b that

$$
\mathbf{U}^{j{ }^{j}{ }_{\mathrm{se} i}}=\mathbf{0}_{12}
$$

while if $j=i$, we find using eq II-22a that

$$
\mathrm{U}^{j \prime}{ }_{\mathrm{sei}}=\left[\begin{array}{lll}
\mathrm{U}_{\mathrm{d}}^{\prime} & \mathrm{U}_{\mathrm{CH}}^{\prime} & 0_{4} \\
\mathbf{0}_{4} & \mathrm{U}_{\mathrm{HH}}^{\prime} & \mathrm{U}^{\prime}{ }^{\prime} \mathrm{HC}, j \\
\mathbf{0}_{4} & \mathbf{0}_{4} & \mathbf{0}
\end{array}\right]_{i}
$$

in which $\mathbf{U}_{\mathrm{d}}^{\prime}, \mathbf{U}_{\mathrm{CH}}^{\prime}$, and $\mathbf{U}_{\mathrm{HH}}^{\prime}$ are given in eq II-10b, II-10e, and II- $10 \mathrm{f}$ and

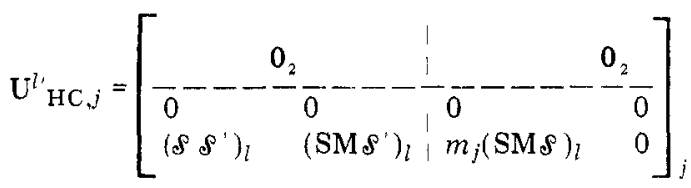

with $\left(\mathcal{S}^{\prime}\right)_{l}$ and $\left(\mathrm{SM}^{\prime}\right)_{l}$ presented in eq II-29c and II-29d, respectively. In addition, $(\mathrm{SM} \mathcal{S})_{l}$ is found in eq II-22d.

If $i<j$, we have

$$
\mathrm{U}^{j^{\prime}}{ }_{\text {sei }}=\left[\begin{array}{lll}
\mathbf{0}_{4} & \mathbf{0}_{4} & \mathbf{0}_{4} \\
\mathbf{0}_{4} & \mathbf{0}_{4} & \mathrm{U}^{\mathbf{l}^{\prime}} \mathrm{HC}, j \\
\mathbf{0}_{4} & \mathbf{0}_{4} & \mathbf{0}_{4}
\end{array}\right]_{i}
$$

wherein

$$
\mathrm{U}^{\mathrm{i}}{ }_{\mathrm{HC}, j}=\left[\begin{array}{c:cc}
\mathbf{0}_{2} & 0_{2} \\
\hdashline \mathbf{0}_{2} & m_{j}\left(\mathrm{SM}_{\mathcal{S}}\right)_{l} & 0
\end{array}\right]_{i}
$$

Case 2: $\boldsymbol{j}=\boldsymbol{N}_{\mathrm{C}}$. If $i<N_{\mathrm{C}}$, it immediately follows from eq II-22a that

$$
\mathbf{U}^{j{ }^{j e} i}{ }=\mathbf{0}_{12}
$$

If $i=j=N_{\mathrm{C}}$, we have

$$
\mathbf{U}^{j \prime}{ }^{j e i}{ }=\mathbf{U}^{\prime}{ }_{\mathrm{seN}}
$$

with $\mathbf{U}_{\mathrm{seN}_{\mathrm{C}}}$ defined in eq II-30a.

If $i>j=N_{\mathrm{C}}$, we find that

$$
\mathrm{U}^{\prime}{ }^{\prime}{ }_{\text {sei }}=\left[\begin{array}{lll}
\mathbf{0}_{4} & \mathrm{U}^{r^{\prime}}{ }^{\prime} \mathrm{CH}, j & 0_{4} \\
\mathbf{0}_{4} & \mathbf{0}_{4} & 0_{4} \\
0_{4} & \mathbf{0}_{4} & \mathbf{0}_{4}
\end{array}\right]_{i}
$$

for which

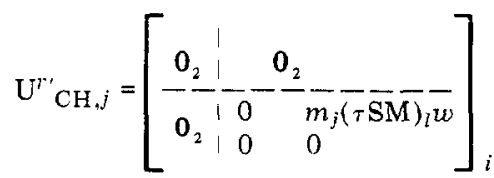

with $j=N_{\mathrm{C}}$.

Case 3: $\boldsymbol{j}>\boldsymbol{N}_{\mathrm{C}}$. In the case that $j>N_{\mathrm{C}}$ and $i \leq N_{\mathrm{C}}$, we have

$$
\mathbf{U}^{j \prime}{ }_{\text {se } i}=\mathbf{0}_{12}
$$

Now suppose $N_{\mathrm{C}}<j<i$; then we have

$$
\mathrm{U}^{j \prime}{ }^{\prime}{ }_{\text {sei }}=\left[\begin{array}{lll}
\mathbf{0}_{4} & \mathrm{U}^{r \prime}{ }^{\prime} \mathrm{CH}, j & \mathbf{0}_{4} \\
\mathbf{0}_{4} & \mathbf{0}_{4} & \mathbf{0}_{4} \\
\mathbf{0}_{4} & \mathbf{0}_{4} & \mathbf{0}_{4}
\end{array}\right]_{i}
$$

with $\mathrm{U}^{\prime \prime}{ }_{\mathrm{CH}, j}$ given by eq II-35d. 
Consider the case with $i=j>N_{\mathrm{C}}$

$$
\mathrm{U}^{j}{ }_{\mathrm{se} i}=\left[\begin{array}{lll}
\mathbf{0}_{4} & \mathrm{U}^{r^{\prime}} \mathrm{CH}_{j} & \mathbf{0}_{4} \\
\mathbf{0}_{4} & \mathrm{U}_{\cdot \mathrm{HH}}^{\prime} & \mathrm{U}^{\prime}{ }_{\mathrm{HC}} \\
\mathbf{0}_{4} & \mathbf{0}_{4} & \mathrm{U}_{\mathrm{d}}^{\prime}
\end{array}\right]_{i}
$$

wherein

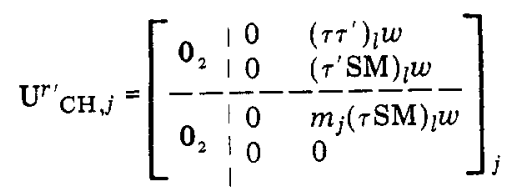

with $\left(\tau \tau^{\prime}\right)_{l},\left(\tau^{\prime} \mathrm{SM}\right)_{l}$, and $(\tau \mathrm{SM})_{l}$ given in eq II-31c, II-31d, and II-26c, respectively. Moreover, $\mathbf{U}^{\prime}{ }_{\mathrm{HH}}, \mathbf{U}_{\mathrm{HC}}^{\prime}$, and $\mathbf{U}_{\mathrm{d}}^{\prime}$ are defined in eq II-10f, II-12b, and II-10b, respectively, with $\mathcal{S}$ and $\tau$ given in eq II-1.

If $j>i$

$$
\mathbf{U}^{j i}{ }_{8 e i}=\mathbf{0}_{12}
$$

This completes the development of the expressions required in the calculation of the helix probability profile.

Fraction of End Coils in Terminal Random Coil Sequences. The fraction of blocks that are both randomly coiled and in end terminal random coil sequences is

$$
f_{\mathrm{ce}}=f_{\mathrm{mce}} \frac{Z_{\mathrm{m}}{ }^{2}}{Z_{\mathrm{c}}}+\left\{\mathbf{J}_{\mathrm{s}} * \prod_{i=1}^{N_{\mathrm{f}}} \mathbf{A}_{\mathrm{fei}} \mathbf{J}_{\mathrm{se}}+\mathbf{J}_{\mathrm{s}} * \prod_{i=1}^{N_{\mathrm{f}}} \mathbf{A}_{\mathrm{bei}} \mathrm{J}_{\mathrm{se}}\right\} /\left(N_{\mathrm{B}} Z_{\mathrm{c}}\right)
$$

$f_{\text {mce }}$ is the fraction of random coils in end random coil sequences in isolated single chains. $\mathbf{J}_{\mathrm{g}}{ }^{*}$ and $\mathbf{J}_{\mathrm{ge}}$ are row and column vectors whose definition may be found following eq II-27.

The $24 \times 24$ supermatrix associated with the unwinding of the random coil blocks from the $\mathrm{N}$ terminus is of the form

$$
\mathbf{A}_{\mathrm{fe} i}=\left[\begin{array}{ll}
\mathrm{U}_{\mathrm{fe}} & \mathrm{U}_{\mathrm{fe}} \\
\mathbf{0}_{12} & \mathrm{U}_{\mathrm{se}}
\end{array}\right]_{i}
$$

with $\mathbf{U}_{\mathrm{ge}}$ given in eq II-22a, II-23a, or II-25 for $i<N_{\mathrm{C}}, i$ $=N_{\mathrm{C}}$, or $i>N_{\mathrm{C}}$, respectively.

If $i<N_{\mathrm{C}}$, we have

$$
\mathrm{U}_{\mathrm{fei}}=\left[\begin{array}{lll}
\mathrm{U}_{\mathrm{fd}} & \mathrm{U}_{\mathrm{fCH}} & \mathbf{0}_{4} \\
\mathbf{0}_{4} & \mathbf{0}_{4} & \mathbf{0}_{4} \\
\mathbf{0}_{4} & \mathbf{0}_{4} & \mathbf{0}_{4}
\end{array}\right]_{i}
$$

with $\mathbf{U}_{\mathrm{fd}}$ and $\mathbf{U}_{\mathrm{fCH}}$ defined in eq II-15c and II-15e, respectively.

If $i=N_{\mathrm{C}}$, we have

$$
\mathrm{U}_{\mathrm{fei}}=\left[\begin{array}{lll}
\mathbf{E}_{4} & \mathrm{U}_{\mathrm{fCH}} & \mathbf{0}_{4} \\
\mathbf{0}_{4} & \mathbf{0}_{4} & \mathbf{0}_{4} \\
\mathbf{0}_{4} & \mathbf{0}_{4} & \mathbf{0}_{4}
\end{array}\right]_{i}
$$

wherein $\mathbf{U}_{\mathrm{fCH}}$ is given by eq II-15e, employing $\tau_{\mathrm{p}}$ of eq II-15f.

If $i>N_{\mathrm{C}}$, we have

$$
\mathrm{U}_{\mathrm{fei}}=\left[\begin{array}{lll}
\mathrm{E}_{4} & \mathrm{U}_{\mathrm{fCH}} & \mathbf{0}_{4} \\
\mathbf{0}_{4} & \mathbf{0}_{4} & \mathbf{0}_{4} \\
\mathbf{0}_{4} & \mathbf{0}_{4} & \mathbf{0}_{4}
\end{array}\right]_{i}
$$

where

$$
\mathbf{U}^{r} r_{\mathrm{fCH}}=\left[\begin{array}{c:cc}
\mathbf{0}_{2} & 0 & \left(\tau_{\mathrm{p}} \tau\right)_{l} w \\
\hdashline 0_{2} & 0 & \left(\tau_{\mathrm{p}} \mathrm{SM}\right)_{l} w \\
\hdashline & \mathbf{0}_{2}
\end{array}\right]_{i}
$$

$$
\begin{gathered}
\left(\tau_{\mathrm{p}} \tau\right)_{l}=\sum_{j=1}^{m_{i}} \sum_{r=1}^{m_{i}}\left[(j-1) / m_{i}\right] \sigma_{j} \sigma_{r} \prod_{\substack{k=j \\
l=r}}^{m_{i}} s_{k} s_{l} \delta\left(2 \sum_{n=N_{\mathrm{C}}+1}^{i-1} m_{n}+\right. \\
\left.2 m_{r}+l_{\mathrm{c}}+j+r-2 ;|j-r|\right) \text { (III-38f) } \\
\left(\tau_{\mathrm{p}} \mathrm{SM}\right)_{l}=\prod_{j=N_{\mathrm{C}}}^{i} \mathrm{SM}_{j} \sum_{r=1}^{m_{i}}\left[\sigma_{r}(r-1) / m_{i}\right] \prod_{k=r}^{m_{i}} s_{k} \delta\left(\sum_{n=N_{\mathrm{C}}+1}^{i-1} m_{n}+\right. \\
\left.m_{r}+l_{\mathrm{c}}+r-1 ; \sum_{n=N_{\mathrm{C}}+1}^{i} m_{n}+m_{r}-\left(m_{i}-r+1\right)\right) \text { (II-38g) }
\end{gathered}
$$

This completes the development of the $\mathbf{A}_{\mathrm{fe}}$-type matrix.

The supermatrix associated with the unwinding of the randomly coiled blocks from the C-terminal end of the molecule is a $24 \times 24$ supermatrix of the form

$$
\mathbf{A}_{\mathrm{be} i}=\left[\begin{array}{ll}
\mathrm{U}_{\mathrm{se}} & \mathbf{U}_{\mathrm{be}} \\
0_{12} & \mathbf{U}_{\mathrm{be}}
\end{array}\right]_{i}
$$

with $\mathbf{U}_{\text {se }}$ defined in eq II-22a, II-23a, or II-25 when $i$ is less than, equal to, or greater than $N_{\mathrm{C}}$, respectively.

When $i<N_{\mathrm{C}}$

$$
\mathrm{U}_{\mathrm{bei}}=\left[\begin{array}{lll}
\mathbf{0}_{4} & \mathbf{0}_{4} & \mathbf{0}_{4} \\
\mathbf{0}_{4} & \mathbf{0}_{4} & \mathrm{U}_{\mathrm{bHC}} \\
\mathbf{0}_{4} & \mathbf{0}_{4} & \mathbf{E}_{4}
\end{array}\right]_{i}
$$

wherein

$$
\mathrm{U}_{\mathrm{bHC}, i}^{l}=\left[\begin{array}{ll:l} 
& \mathbf{0}_{2} & \mathbf{0}_{2} \\
\hdashline 0 & 0 & \mathbf{0}_{2} \\
\left.\hdashline \mathcal{S}_{\mathrm{p}}\right)_{l} & \left(\mathrm{SM}_{\mathrm{p}}\right)_{l}
\end{array}\right] ;
$$

and

$$
\begin{aligned}
& \left(\mathcal{S}_{\mathrm{p}}\right)_{l}=\delta\left(2 \sum_{j=i}^{N_{\mathrm{C}}-1} m_{i}+2 m_{l}+l_{\mathrm{c}} ; 0\right)+\sum_{r=1}^{m_{i}-1}(1+ \\
& \left.\frac{m_{i}-r}{m_{i}}\right) \prod_{k=1}^{r} s_{k} \delta\left(2 \sum_{j=i}^{i-1} m_{j}+2 m_{l}+l_{\mathrm{c}}-r ; r\right)+\sum_{n=1}^{m_{l}-1} \sum_{r=1}^{m_{r}-1}\left[\left(m_{i}-\right.\right. \\
& \left.r) / m_{i}\right] \prod_{k=1}^{n} s_{k} \prod_{l=1}^{r} s_{r} \delta\left(2 \sum_{j=i}^{N_{\mathrm{C}^{-1}}} m_{j}+2 m_{l}+l_{c}-n-r ;|n-r|\right)
\end{aligned}
$$

$$
\begin{aligned}
& \left(\mathrm{SM}_{\mathrm{p}}\right)_{l}= \\
& \prod_{j=i}^{N_{C^{-1}}} \mathrm{SM}_{j}\left[\delta\left(\sum_{k=i}^{N_{\mathrm{C}^{-1}}} m_{k}+m_{l}+l_{\mathrm{c}} ; \sum_{k=i}^{N_{\mathrm{C}}-1} m_{k}+m_{l}\right)+\sum_{r=1}^{m_{-}-1}\left[\left(m_{i}-\right.\right.\right. \\
& \left.\left.r) / m_{i}\right] \prod_{k=1}^{r} s_{k} \delta\left(\sum_{j=i}^{N_{\mathrm{C}}-1} m_{j}+m_{l}+l_{\mathrm{c}}-r ; \sum_{j=i}^{N_{\mathrm{C}}-1} m_{j}+m_{l}-r\right)\right]
\end{aligned}
$$

If $i=N_{\mathrm{C}}$,

$$
\mathrm{U}_{\mathrm{be} i}=\left[\begin{array}{lll}
\mathbf{0}_{4} & \mathbf{0}_{4} & \mathbf{0}_{4} \\
\mathbf{0}_{4} & \mathbf{0}_{4} & \mathrm{U}_{\mathrm{bHC}}^{l} \mathrm{U}_{N_{\mathrm{C}}} \\
\mathbf{0}_{4} & \mathbf{0}_{4} & \mathrm{U}_{\mathrm{b} N_{\mathrm{C}}}
\end{array}\right]_{N_{\mathrm{C}}}
$$

in which $\mathrm{U}_{\mathrm{bHC}}^{l}$ is of the same form as eq II-39c, but with

$$
\begin{gathered}
\left(\mathcal{S}_{\mathrm{p}}\right)_{l}=\delta\left(2 m_{l}+l_{c} ; 0\right) \\
\left(\mathrm{SM} \mathcal{S}_{\mathrm{p}}\right)_{l}=\delta\left(m_{l}+l_{c} ; m_{l}\right)
\end{gathered}
$$

Furthermore

$$
\mathrm{U}_{\mathrm{b} N_{\mathrm{C}}}=\left[\begin{array}{llll}
1 & 0 & 0 & 0 \\
0 & \mathrm{SM}_{N_{\mathrm{C}}} & 0 & 0 \\
0 & 0 & 0 & 0 \\
0 & 0 & 0 & 0
\end{array}\right]
$$




$$
\mathbf{U}_{\mathrm{bei}}=\mathbf{U}_{\mathrm{bsi}}
$$

with $\mathrm{U}_{\mathrm{bsi}}$ defined in eq II-18a.

Summarizing the results of section II, we have developed expressions for the partition function, the overall helix content, the helix probability profile, and the fraction of random coil blocks in terminal random coil sequences for the loops-excluded and interior eyelet model of a twochain, coiled coil containing a single cross-link. In the former model, there are no interior random coil loops whatsoever between interacting pairs of $\alpha$-helices. In the latter model, interior random coil loops that terminate on interacting pairs of helices are allowed to originate at the cross-link, resulting in the possibility of states containing one or two random coil eyelets in addition to those states included in the loops-excluded model.

\section{Application to Homopolypeptides}

The first point to be addressed is the difference in helix content calculated, at a given value of the helix-helix interaction parameter, in the loops-excluded model as compared to the interior eyelet model of singly cross-linked, two-chain, coiled coils (dimers). Since the interior eyelet model includes additional states of lower helix content, it follows at a given $w$ that $Z_{\text {int }} \geq Z_{\text {int }}{ }^{0}$ and $f_{\text {he }}{ }^{0} \geq f_{\text {he }}$ (the presence (absence) of a superscript zero refers to an expression calculated via the loops-excluded (interior eyelet) model). We would expect type I and II interior random coil loops to be most important when the cross-link occurs in an end block and for a given $s$, when $\sigma$ is small. The latter conditions obtains because at fixed $s$ the helix content is lower when $\sigma$ is smaller at $w=1.0$, so that interior random coil states are proportionately more important. In the limit of high helix content, the helix content of the cross-linked dimer vs. $w$ curve should converge to the non-cross-linked, two-chain, coiled coil limit.

In all cases discussed below unless otherwise stated, we have set $m=4, m_{l}=2, m_{r}=3, l_{c}=2, s=0.94$, and $u_{\phi}=$ $359 \AA^{3} \cdot{ }^{14}$

In the following, we examine two illustrative cases, one in the limit of very small $\sigma$ and the other in the limit of fairly large values of $\sigma$. In particular, we shall focus on a 284 residue per chain, homopolymer dimer divided into $N_{\mathrm{B}}=71$ blocks, containing four residues per block with $N_{\mathrm{C}}=1$. The helix content of the subpopulation of the cross-linked dimer containing at least one interacting pair of helices in the loops-excluded (interior eyelet) model, $f_{\mathrm{d}}{ }^{0}$, $\left(f_{d}\right)$ is obtained by using eq II-8b (II-27b). Setting $\sigma=10^{-6}$, if $w=1.0, f_{\mathrm{d}}{ }^{0}=0.0676303637$, which $f_{\mathrm{d}}=0.0676295079$. (For $w$ on the order of unity, the noninteracting conformation makes the overwhelming contribution to the overall helix content, and since $f_{\mathrm{hm}}$ is the same for the two models, the more sensitive comparison is between $f_{\mathrm{d}}{ }^{0}$ and $f_{\mathrm{d}}$ ). Taking $w=1.60$, we find $f_{\mathrm{d}}{ }^{0}=0.380595834$ and $f_{\mathrm{d}}=$ 0.380589991 . In fact, over the entire $f_{\mathrm{d}}{ }^{0}$ or $f_{\mathrm{d}}$ vs. $w$ curve, the two models give helix contents that agree to at least four significant figures and in limit that $w \rightarrow \infty$, the two curves coalesce. Consider now the case with $\sigma=10^{-2}$. If $w=1.0, f_{\mathrm{d}}{ }^{0}=0.358852889$ and $f_{\mathrm{d}}=0.358852088$. On increasing $w$ to $2.5, f_{\mathrm{d}}{ }^{0}=0.528992096$ and $f_{\mathrm{d}}=$ 0.528991175 . On the basis of these extreme cases as well as for a large number of calculations as a function of $\sigma, N_{\mathrm{B}}$, and $N_{\mathrm{C}}$ not displayed here, it is apparent that the helix contents given by the two models are indistinguishable. Thus we conclude that the net effect of loop entropy is so prohibitive as to preclude the formation of interior random coiled states at the cross-link. In other words, in molecules containing an interacting helical stretch, the interacting helical stretch essentially always includes the cross-linked

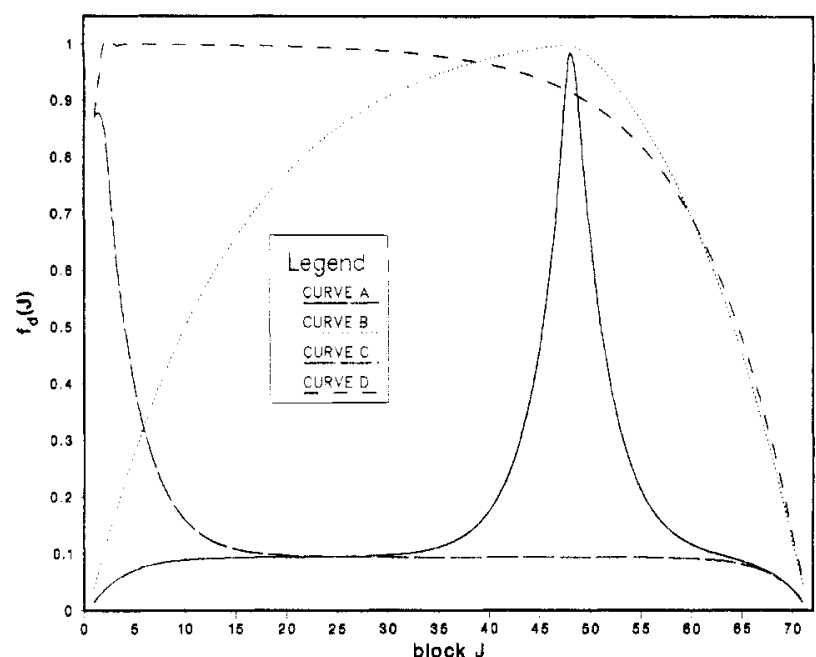

Figure 1. Helix content of the $j$ th block for cross-linked molecules containing an interacting helical stretch, $f_{\mathrm{d}}(j)$ vs. $j$ calculated by employing eq II-32 for a homopolymeric, two-chain, coiled coil with $N_{\mathrm{B}}=71, m=4, m_{l}=2, m_{r}=3, l_{\mathrm{c}}=2, s=0.94$, and $\sigma=$ $5 \times 10^{-4} . N_{\mathrm{C}}=48(1)$ and $w=1.0(1.0)$ and 1.85 (1.90) in curves $A$ and $B(C$ and $D)$, respectively.

block (see Figure 1). This is entirely consistent with our previous work on non-cross-linked chains where we concluded there is just a single interacting helical stretch in the dimer. ${ }^{2}$

The origin of the enhanced helical stability of the cross-linked block is due entirely to the destabilization of type I and II interior random coil stretches that originate at the cross-link and terminate at the beginning of the interacting helical stretch. Even in the absence of interhelical stabilization, i.e., if $w=1.0$, the statistical weight $\delta$ of the minimum length type I random coil state calculated via eq II-20a and containing six residues is 0.016 , whereas the statistical weight of the fully helical pair of blocks is 0.61 . The statistical weight $\delta$ of the minimum length type II random coil state containing four residues and calculated via eq II-20a is 0.028 , whereas the statistical weight of the fully helical block is 0.78 . In other words, such interior random coil loops are extremely unlikely. The preceding calculation neglects the statistical weight of $\mathrm{C}[\mathrm{H}]$ states and the statistical weight of the cross-link itself. This leads us to conjecture that out-of-register states that by necessity must contain (type I or type II) constrained interior random coil loops can probably be neglected. We shall examine the importance of out-of-register states in a forthcoming paper.

If the cross-linked block is located somewhere in the middle of the chain, so that initiating [C]H-type states of the interating helical stretch contribute negligibly, then $f_{\mathrm{d}}\left(N_{\mathrm{C}}\right)$ will be independent of the statistical weight of the helical conformation of the cross-linked block; i.e., it is insensitive to $\sigma, s$, and $w$ of the cross-linked block. As the cross-linked block moves toward the chain ends, $f_{\mathrm{d}}\left(N_{\mathrm{C}}\right)$ is a weighted average over the $[\mathrm{C}] \mathrm{H}$ and $[\mathrm{H}] \mathrm{H}$ conformations of the block and equals the ratio $\tau^{\prime} / m \tau$ when $N_{\mathrm{C}}=1$. Thus, there is a range of values of $N_{\mathrm{C}}$ near the chain ends where $f_{\mathrm{d}}\left(N_{\mathrm{C}}\right)$ is in principle a function of $\sigma$. In practice, for the cases examined, where $N_{\mathrm{B}}=71$ and $l \leq N_{\mathrm{C}} \leq 10$, we found that $f_{\mathrm{d}}\left(N_{\mathrm{C}}\right)$ changes by about $1 \%$ on increasing $\sigma$ from $5 \times 10^{-4}$ to $10^{-2}$ when $w=1.0$. A smaller change in $f_{\mathrm{d}}\left(N_{\mathrm{C}}\right)$ is expected as $w$ gets larger. Hence we conclude that the helix content of the cross-linked block pair is in practice a very insensitive function of $\sigma$.

The above statements are verified in Figure 1, where we plot $f_{d}(j)$ vs. block number $j$ calculated by emplying eq II-32 for the homopolymeric analogue of tropomyosin with 
$N_{\mathrm{B}}=71, N_{\mathrm{C}}=48, \sigma=5 \times 10^{-4}$, and $w=1.0$, with $f_{\mathrm{d}}=$ 0.1752 and $f_{\text {he }}=0.08552$, and $w=1.85$, with $f_{\mathrm{d}}=0.7047$ and $f_{\text {he }}=0.5810$, in curves $A$ and $B$, respectively. When $w=1.0, f_{\mathrm{d}}(48)=0.9834$, and when $w=1.85, f_{\mathrm{d}}(48)=$ 0.9999 . In Figure 1, curves $\mathrm{C}$ and $\mathrm{D}$, we have also set $N_{\mathrm{B}}$ $=71, N_{\mathrm{C}}=1, \sigma=5 \times 10^{-4}$, and $w=1.0$, with $f_{\mathrm{d}}=0.1408$ and $f_{\mathrm{he}}=0.08546$, and $w=1.90$, with $f_{\mathrm{d}}=0.8492$ and $f_{\mathrm{he}}$ $=0.1373$, respectively. Observe that the cross-link $f_{\mathrm{d}}(1)$ $=0.87098$ when $w=1.0, f_{d}(1)=0.87102$ when $w=1.92$, and $f_{\mathrm{d}}(1)=0.87108$ when $w=3.0$. Moreover, the value is insensitive to the value of $\sigma$. If we set $w=1.0$ and take $\sigma=10^{-2}, f_{\mathrm{d}}(1)=0.87107$. While the $f_{\mathrm{d}}(j)$ changes as a function of $w$ and $\sigma$ for $j>1$, the helix content of the cross-linked end block is insensitive to both $\sigma$ and $w$ and reflects the dominant role played by loop entropy. The helix content at the cross-linked block is less than unity, since residues 1 to $m_{l}$ may be randomly coiled. In fact, if we neglect the possiblity of closed random coil loops and assert that the helix content of block one is simply $\tau^{\prime} / m \tau$ we find $f_{\mathrm{d}}(1)=0.871134$, which is the result obtained in the loops-excluded model. Moreover, the above result is insensitive to $N_{\mathrm{B}}$; for example, if $N_{\mathrm{B}}=26, \sigma=5 \times 10^{-4}$, and $N_{\mathrm{C}}=1, f_{\mathrm{d}}(1)=0.87098$ when $w=1.0$ and $f_{\mathrm{d}}(1)=$ 0.87108 when $w=3.0$. The above discussion clearly illustrates the effect of loop entropy in prohibiting interior random coil loops between interacting helical stretches.

If $\tau$ and SM of the cross-linked block are modified due to the possibility of stress at the cross-link, the helix content of the subpopulation composed of noninteracting states, $f_{\mathrm{hm}}$, may change. If $\sigma$ and $s$ of the cross-linked residue are decreased, $f_{\mathrm{hm}}$ will decrease. However, since the modification is presumably localized, $f_{\mathrm{hm}}$ is likely to remain within experimental error of the non-cross-linked, single-chain helix content.

This is not to say, however, that the experimentally determined overall helix content $f_{\text {he }}$ is independent of $\sigma$, $s$, and $w$ of the cross-linked residue. If there is a stress localized at the cross-link pair of blocks alone, then, while $f_{\text {hm }}$ and $f_{\mathrm{d}}$ remain essentially unchanged, $f_{\text {he }}$ may change since it is a weighted average of interacting and noninteracting conformations. Modifications in $\sigma, s$, and $w$ of the cross-linked blocks will act to shift the relative equilibrium between interacting and noninteracting states. Thus, the net effect will be to modify the apparent value of $r_{\phi}$ extracted from experiment (see eq II-21c). For cross-links located at $N_{\mathrm{C}}>1$ and in interacting conformations, the cross-linked block is essentially fully helical. Thus, the apparent $r_{\phi}$ is the product of two factors: (a) the ratio of the statistical weight of the fully helical, cross-linked pair of blocks to the fully helical, non-crosslinked block pair $\left(\mathrm{SM}^{2} w\right)$ and (b) the correct $r_{\phi}$, that is, the ratio, $u_{\phi} / v_{\phi}$. If, however, the stress is not localized to the cross-link pair of blocks, then $f_{\mathrm{d}}$ will change. The magnitude of the decrease in $f_{\mathrm{d}}$ will of course depend on the size of the distorted region in the $\alpha$-helices introduced by the cross-link.

The next point we address is the range of physically reasonable values for $r_{\phi}$. Recent theoretical and experimental work on the two-chain, coiled coil tropomyosin provides some qualitative guidance. ${ }^{35}$ For the entire range of temperatures studied, the theoretically calculated helix content of a non-cross-linked dimer that contains at least one interacting helical stretch, $f_{\text {hd }}$, always exceeds the experimentally measured helix content of the cross-linked molecule, $f_{\mathrm{he}}{ }^{35}$ It should be pointed out that cross-linked tropomyosin seems to undergo a pretransition with concomitant loss of helix content before the final, very steep main transition occurs. . $6,36,37$ This pretransition may be

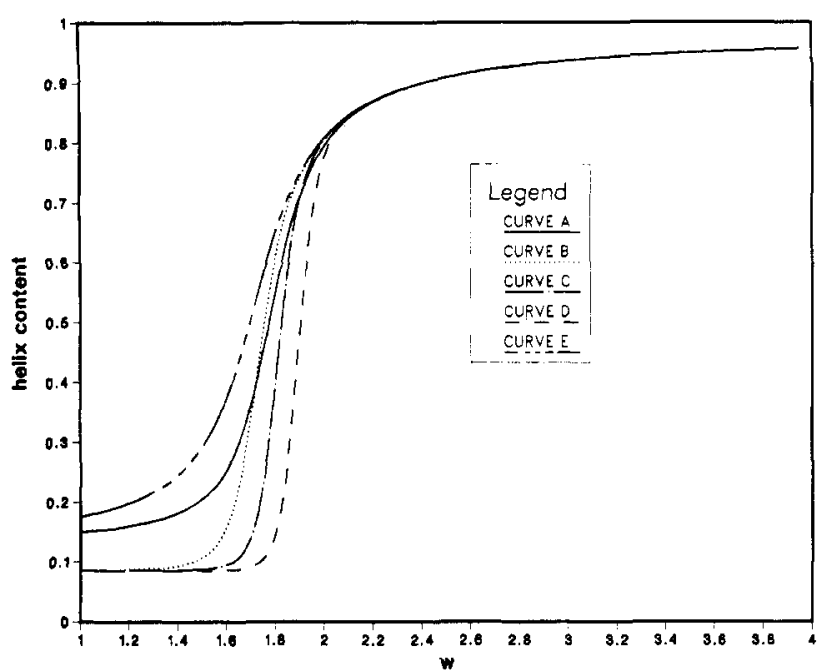

Figure 2. Curve A: plot of the helix content of the subpopulation of a non-cross-linked, homopolymeric, two-chain, coiled coil in the perfect-matching limit, containing at least a pair of interacting $\alpha$-helical turns, $f_{\text {hd }}$, and calculated via eq II-10 of ref 3 vs. the helix-helix interaction parameter $w$. Curves B-D: plots of the overall helix content for a cross-linked, homopolymeric, two-chain, coiled coil calculated via eq II-27b, $f_{\text {he }}$, vs. $w$ with $r_{\phi}=1.0,10^{-1}$, and $10^{-2}$, respectively. Curve E: plot of $f_{\mathrm{d}}$, the helix content of the subpopulation of cross-linked chains containing at least one interacting pair of helical blocks calculated via eq II-27b, vs. $w$. In all cases, $N_{\mathrm{B}}=71, m=4, s=0.94$, and $\sigma=5 \times 10^{-4}$ and for cross-linked chains, $N_{\mathrm{C}}=48, m_{l}=2, m_{r}=3$, and $l_{\mathrm{c}}=2$.

due to the difference in stability of the N-terminal half vs. the C-terminal half of tropomyosin ${ }^{38-41}$ as well as stress induced in the vicinity of the cross-linked block and cannot be mimicked by a homopolymeric cross-linked dimer with a uniform, site-independent $w$.

To estimate the magnitude of $r_{\phi}$, in Figure 2 we have plotted in curve $A$ the helix content of the subpopulation of a non-cross-linked dimer that contains at least one interacting pair of $\alpha$-helical blocks, $f_{\text {hd }}$, vs. $w$ for a homopolymeric two-chain, coiled coil in the perfect-matching limit with $N_{\mathrm{B}}=71$ and $\sigma=5 \times 10^{4}$, calculated by employing eq II-10 of ref 3 . (The internal partition function of the non-cross-linked dimer $Z_{\text {sd }}$ in eq II-8 of ref 3 contains the incorrect quantity $2 Z_{m}-1$, which should be eliminated; similarly, $f_{\mathrm{hd}}$ in eq II-10 of ref 3 contains the term $f_{\mathrm{hm}} Z_{\mathrm{m}} / Z_{\text {sd }}$, which should be ignored.) We have also plotted $f_{\text {he }}$ vs. $w$ calculated by employing eq II-27b for a crosslinked, homopolymeric, two-chain, coiled coil having $N_{\mathrm{B}}$ $=71, N_{\mathrm{C}}=48$, and $\sigma=5 \times 10^{-4}$ with $r_{\phi}=1.0,10^{-1}$, and $10^{-2}$ in curves B-D, respectively. Thus, we have the homopolymeric analogue of tropomyosin that is cross-linked at residue 190 (residue 190 in rabbit $\alpha$-tropomyosin contains a pair of cysteine residues that can be cross-linked).

In Figure 2, curve B, with $r_{\phi}=1.0$, crosses the noncross-linked dimer curve (curve $A$ ) in the vicinity of $40 \%$ helix content, in qualitative disagreement with the behavior seen in tropomyosin. Hence, not surprisingly, $r_{\phi}$ must be less than unity. Curve $\mathrm{C}$, with $r_{\phi}=10^{-1}$, slightly rises above curve $\mathrm{A}$ in the vicinity of $70 \%$ helix content, but such a crossing would lie within experimental error. Curve $\mathrm{D}$, with $r_{\phi}=10^{-2}$, always lies below curve $\mathrm{A}$, with the two curves coalescing in the vicinity of $80 \%$ helix content.

In the calculations discussed below, to be conservative, we have taken $r_{\phi}=0.1$, a value that is essentially in qualitative accord with some of the combined theoretical and experimental results for tropomyosin. For example, the steepness of $f_{\text {he }}$ vs. $w$ curves is in qualitative agreement with the behavior seen in the main thermal transition of 
cross-linked tropomyosin. As mentioned above, however, no pretransition is evident.

We should point out that the qualitative behavior discussed below is independent of the particular value of $r_{\phi}$. In practice, of course, $r_{\phi}$ must be fit to experiment. For consistency we have presented $f_{\text {hd }}$ vs. $w$ curves calculated in the perfect-matching limit. Inclusion of out-of-register states will tend to uniformly decrease $f_{\text {hd }}$ and further reinforce the conclusion that $r_{\phi}$ is less than unity.

It has been stated at various times in the literature that the stabilities of cross-linked and non-cross-linked dimers are different, with the main transition being more ${ }^{16}$ $\left(\right.$ less $^{37,42}$ ) stable in the former than in the latter. Actually, care should be exercised in the comparison of the various populations. The experimentally observed helix content of a non-cross-linked, two-chain, coiled coil

$$
\Phi_{\mathrm{h}}=g_{\mathrm{m}} f_{\mathrm{hm}}+\left(1-g_{\mathrm{m}}\right) f_{\mathrm{hd}}
$$

is concentration dependent since the weight fraction of chains that exist as isolated single chains or monomers, $g_{\mathrm{m}}$, is concentration dependent. Observe that the quantities $f_{\mathrm{hm}}$ and $f_{\mathrm{hd}}$ and the partition functions of the single chains and the dimer are concentration independent.

Clearly, we want to compare the helix content of the subpopulation containing at least one interacting pair of helical turns, $f_{\text {hd }}$, with the cross-linked chain, as was done in Figure 2, curves A-D. However, $f_{\text {he }}$ contains a contribution from the noninteracting states, $f_{\mathrm{hm}}$ as well as the interacting conformations, $f_{\mathrm{d}}$; viz.

$$
f_{\mathrm{he}}=\frac{Z_{m}{ }^{2}}{Z_{\mathrm{c}}} f_{\mathrm{hm}}+\frac{r_{\phi} Z_{\text {int }}}{Z_{\mathrm{c}}} f_{\mathrm{d}}
$$

In fact, since the cross-linked and interacting subpopulation is essentially always helical at the cross-link, $f_{\mathrm{d}}>f_{\mathrm{hd}}$. This is verified in Figure 2, curve E, where $f_{\mathrm{d}}$ vs. $w$ calculated by employing eq $\mathrm{II}-27 \mathrm{~b}$ is plotted for the same homopolymeric, cross-linked dimer as was considered in curves B-D. Observe that $f_{\mathrm{d}}$ is independent of $r_{\phi}$. Thus, due entirely to loop entropy, cross-linked two-chain, coiled coils containing an interacting helical stretch are predicted to be more stable than the non-cross-linked dimer under identical conditions, that is, at the same $\sigma, s$, and $w$.

We should also point out that for the value of $w$ where $f_{\mathrm{d}}$ contributes appreciably to $f_{\mathrm{he}}$, the cross-linked block at $N_{\mathrm{C}}=48$ is in the middle of an interacting helical stretch. Thus the interchain initiation helix parameter $u_{\phi}$ (see discussion in section IIA) is not solely a property of the cross-linked block in particular but rather represents a value averaged over all helical blocks (we have assumed $r_{\phi i}$ to be site independent).

In Figure 3, we have plotted $f_{\text {he }}$ vs. $w$ calculated via eq II-27b for a homopolymeric, cross-linked, two-chain, coiled coil having $N_{\mathrm{B}}=71, N_{\mathrm{C}}=36, r_{\phi}=0.1$, and $\sigma=10^{-6}, 10^{-5}$, $10^{-4}, 10^{-3}$, and $10^{-2}$ in curves $\mathrm{A}-\mathrm{E}$, respectively. The qualitative behavior as a function of $\sigma$ is typical of twochain, coiled coils and is independent of the presence or location of the cross-link. ${ }^{2-4}$ In the limit of small $\sigma$, the only appreciable helix content is due to the single interacting pair of helices. Increasing $\sigma$ decreases the minimum value of $w$ necessary for the interacting helical states to dominate the population. As $\sigma$ is increased further, the noninteracting conformations begin to have an appreciable helix content, as well as a proportionately larger contribution, $Z_{\mathrm{m}}{ }^{2}$, to the total partition function, $Z_{\mathrm{c}}$. Thus, $w$ must again increase so that interacting helical states, embodied in the $r_{\phi} Z_{\text {int }}$ term, begin to contribute significantly to the population (see eq II-21c). In all cases, in the limit of very high helix, the curves having larger values of $\sigma$ approach those having smaller values of $\sigma$ from below.

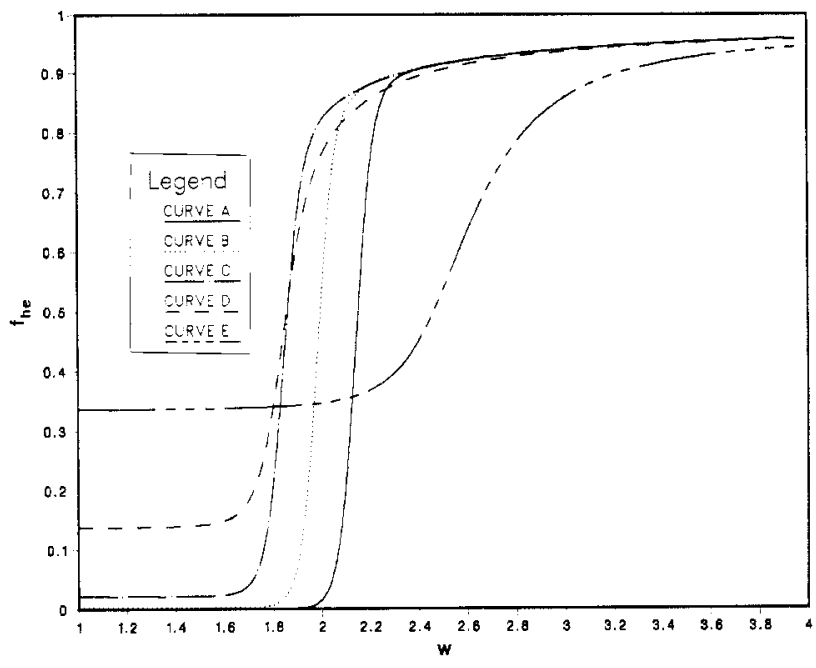

Figure 3. Plot of the overall helix content of a cross-linked, homopolymeric, two-chain, coiled coil, $f_{\text {he }}$, calculated via eq II-27b vs. $w$ with $N_{\mathrm{B}}=71, N_{\mathrm{C}}=36, m=4, m_{l}=2, m_{r}=3, l_{\mathrm{c}}=2, s=$ $0.94, r_{\phi}=10^{-1}$, and $\sigma=10^{-6}, 10^{-5}, 10^{-4}, 10^{-3}$, and $10^{-2}$ in curves A-E, respectively.

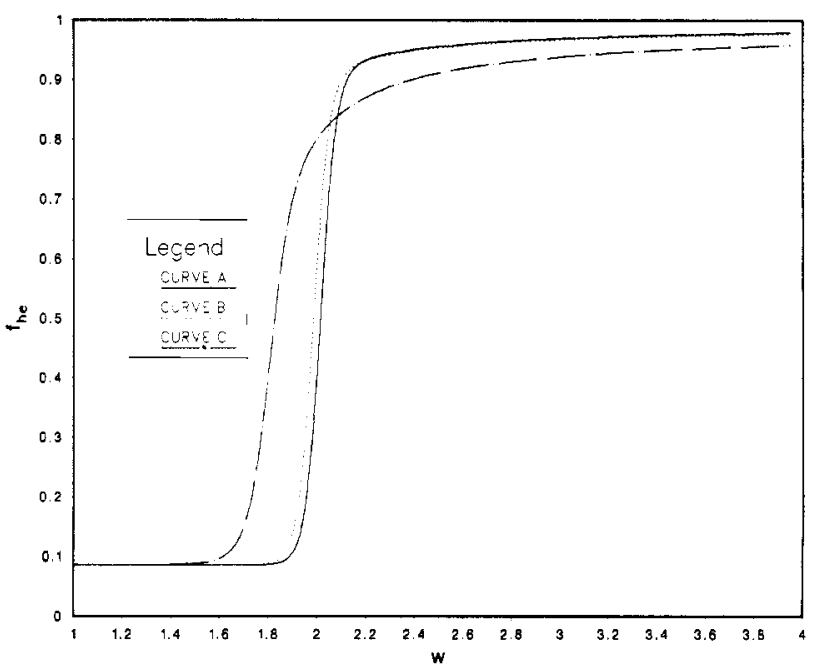

Figure 4. Plot of the overall helix content of a cross-linked, homopolymeric, two-chain, coiled coil, $f_{\text {he, }}$, calculated via eq II- $27 \mathrm{~b}$ vs. $w$ with $N_{\mathrm{B}}=71, m=4, m_{l}=2, m_{r}=3, l_{\mathrm{c}}=2, s=0.94, \sigma=$ $5 \times 10^{-4}, r_{\phi}=10^{-1}$, and $N_{\mathrm{C}}=71,1$, and 36 in curves A-C, respectively.

We next examine the sensitivity of the helix content to the location of the cross-link. In Figure 4 we have plotted $f_{\text {he }}$ vs. $w$ obtained via eq II-27b for a homopolymeric, cross-linked, two-chain, coiled coil having $N_{\mathrm{B}}=71, \sigma=5$ $\times 10^{-4}, r_{\phi}=0.1$, and $N_{\mathrm{C}}=71,1$, and 36 in curves A-C, respectively. That is, we have placed the cross-link at either of the two ends or in the middle of the cross-linked dimer. The difference between curves $A$ and $B$ is an artifact of the present method of calculation, in which we assume that the cross-link is completely randomly coiled if it is part of an $[\mathrm{H}] \mathrm{C}$-type state but not if it is part of a [C]H state, where interfacial helical states are allowed. Curves $\mathrm{A}$ and $\mathrm{B}$ are qualitatively different from curve $\mathrm{C}$; the former two transitions are far more cooperative than the latter. Basically, the cross-linked block is completely helical on the side of the cross-link nearest to the middle of the chain; thus the single interacting helical stretch giving rise to the augmented stability must originate from the chain end that contains the cross-link, and there is a severe reduction in the number of states containing interacting helices (of order $N_{\mathrm{B}} / 4$ ) relative to the case where the cross-link lies near the middle of the chain. For all 


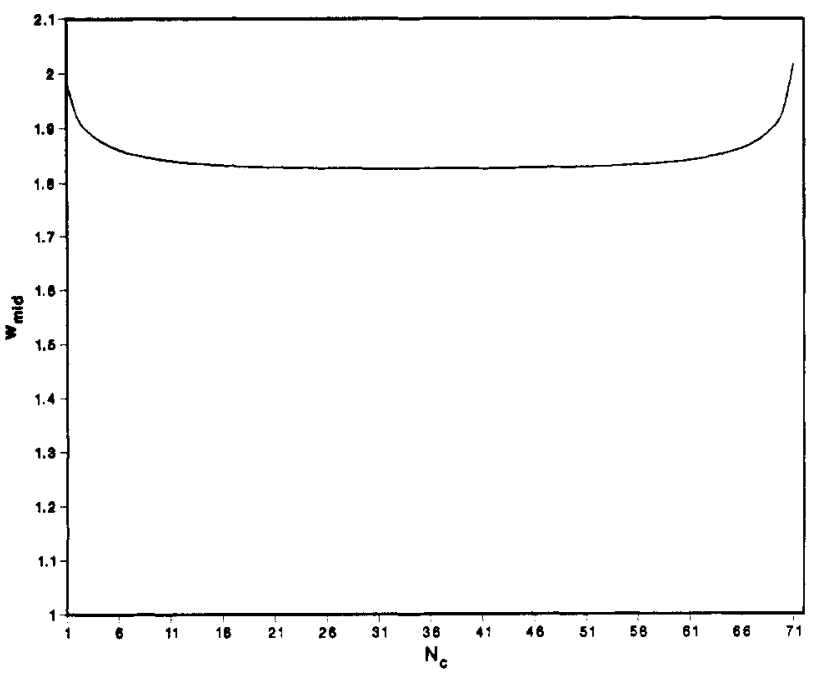

Figure 5. Plot of the value of the helix-helix interaction parameter, $w_{\text {mid }}$, necessary for $f_{\text {he }}$ to be equal to $50 \%$ vs. the location of the cross-linked block, $N_{\mathrm{C}}$.

values of $w$, then, $Z_{\text {int }}\left(N_{\mathrm{C}}=36\right) \geq Z_{\text {int }}\left(N_{\mathrm{C}}=1\right)$ or $Z_{\text {int }}\left(N_{\mathrm{C}}\right.$ $=71$ ), and at lower values of $w$, because of the inequality of the partition functions $f_{\mathrm{d}}\left(N_{\mathrm{C}}=36\right) \geq f_{\mathrm{d}}\left(N_{\mathrm{C}}=1\right)$ or $f_{\mathrm{d}}\left(N_{\mathrm{C}}\right.$ $=71)$. At high helix content, there are now more ways of inserting random coil states when $N_{\mathrm{C}}=36$ than when $N_{\mathrm{C}}$ $=1$ or 71 ; hence the $f_{\mathrm{d}}$ vs. $w$ (and equivalently the $f_{\text {he }}$ vs. $w)$ curves must cross. Thus, chains that are cross-linked at the ends experience a more cooperative transition than chains that are cross-linked near the middle (i.e., their effective $\sigma$ has decreased). This purely entropic effect emerges as a consequence of the continuous nature of the helix-coil transition.

It is of interest to examine the sensitivity of the helixcoil transition in cross-linked chains to the location of the cross-link. In Figure 5, we have plotted $w_{\text {mid }}$, the value of the helix-helix interaction parameter necessary for $f_{\text {he }}$ to be equal to $50 \%$, vs. $N_{\mathrm{C}}$. Clearly, for values of $6 \leq N_{\mathrm{C}} \leq$ $65, w_{\text {mid }}$ is as a practical matter insensitive to the location of the cross-link. In other words, the theory predicts that cross-linked chains with $6 \leq N_{\mathrm{C}} \leq 65$ should have essentially the same thermal denaturation profiles. (Rabbit $\beta$-tropomyosin is cross-linkable at residues 36 and 190.) However, the thermal denaturation profile should change drastically if the cross-link is placed in the first or second pair of $\alpha$-helical turns nearest the ends. Thus, it would be of interest to attempt to experimentally cross-link tropomyosin at one of the chain ends and compare the thermal denaturation profile with a tropomyosin molecule cross-linked at cysteine-190.

Finally, to investigate the dependence of the $f_{\text {he }}$ on degree of polymerization, in Figure 6 we have plotted $f_{\text {he }}$ vs. $w$ calculated via eq II-27b for a homopolymeric two-chain, coiled coil having $N_{\mathrm{B}}=26, \sigma=5 \times 10^{-4}, r_{\phi}=10^{-1}$, and $N_{\mathrm{C}}$ $=26,1$, and 13 in curves A-C, respectively. As expected, the transition curves are broader in Figure 6 when $N_{\mathrm{B}}=$ 26 than in Figure 5, where $N_{\mathrm{B}}=71$. Moreover, the sensitivity to the location of the cross-link has increased; i.e., the $w_{\text {mid }}$ is about 2.25 when $N_{\mathrm{C}}=13$, as compared to $w_{\text {mid }}$ $=2.60$ when $N_{\mathrm{C}}=1$. Basically, when the degree of polymerization is less, there are a smaller number of helical states that can occur. This fact produces a proportionately larger $w_{\mathrm{m}} i_{\mathrm{d}}$ required to achieve $f_{\text {he }}=0.50$ for the cross-link located at the chain ends, as compared to the middle of the chain when $N_{\mathrm{B}}=26$ relative to the case when $N_{\mathrm{B}}=$ 71.

The fraction of interior random coils $f_{\mathrm{ce}}$ vs. $w$ (eq II-37) behaves qualitatively similar to the non-cross-linked case ${ }^{3}$

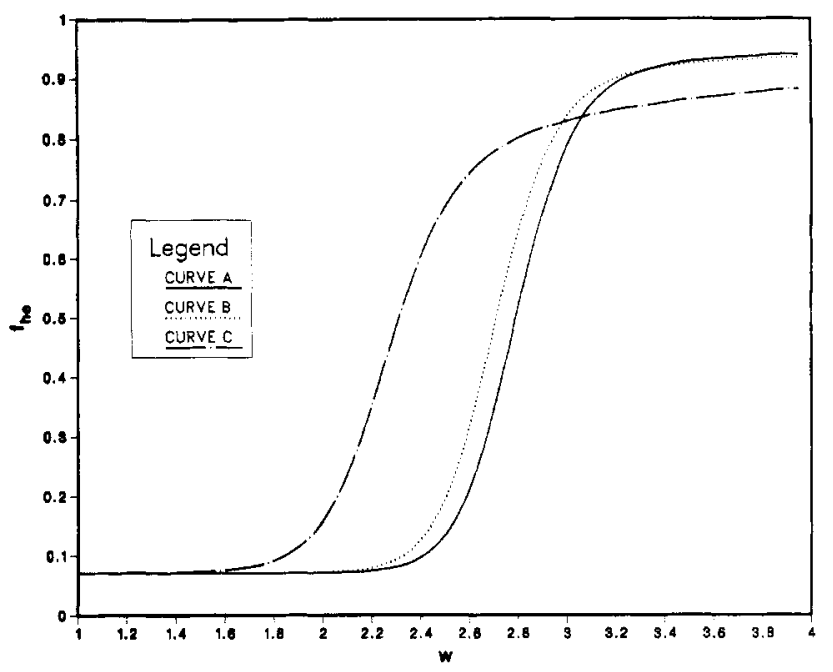

Figure 6. Plot of the overall helix content of a cross-linked, two-chain, coiled coil, $f_{\text {he, }}$ calculated via eq II-27b with $N_{B}=26$, $m=4, m_{l}=2, m_{r}=3, l_{\mathrm{c}}=2, s=0.94, \sigma=5 \times 10^{-4}, r_{\phi}=10^{-1}$, and $N_{\mathrm{C}}=26,1$, and 13 in curves A-C, respectively.

and so is not displayed here.

In summary, we have applied the loops-excluded and interior eyelet models of the helix-coil transition to homopolymeric, singly cross-linked, $\alpha$-helical, two-chain, coiled coils. In the loops-excluded model, interior random coil loops originating at the cross-link and terminating at an interacting helical stretch are a priori excluded. In the interior eyelet model, all such type I and II interior random coil loops are permitted but due to the effect of loop entropy pay a heavy entropic price. We have demonstrated that over a wide variety of conditions the interior eyelet model reduces to the loops-excluded model. Although interior random coiled loops are not a priori excluded, their net contribution to the partition function is sufficiently small that they can be safely ignored. The consequence of this is that there is just a single pair of interacting helices per molecule that includes the cross-linked block, and in this subpopulation of states the cross-linked block will tend to be very highly helical. Furthermore, we have examined the physically reasonable range of the parameter $r_{\phi}$, which is the ratio of the effective volume accessible to an interacting helical block when the helical block in the other chain is held fixed to the effective volume accessible to one of the two noninteracting and cross-linked blocks when the other block is held fixed. Values of $r_{\phi}$ on the order of 0.1 or less seem to be in qualitative agreement with trends seen in singly cross-linked tropomyosin. At a given degree of polymerization, the overall helix content vs. $w$ curve is fairly insensitive to the location of the cross-link provided the cross-link is not located too close to the chain ends. However, the theory predicts a strikingly enhanced cooperativity for chains cross-linked at the ends relative to chains cross-linked near the middle. This is an important qualitative prediction that requires verification by experiment. Thus, the theory of the helix-coil transition of singly cross-linked, two-chain, coiled coils has been put on an equal footing with the theory for non-cross-linked chains.

\section{Discussion}

In the present work, the loops-excluded and interior eyelet models of the helix-coil transition have been applied to a hypothetical homopolymeric, two-chain, coiled coil. The following important qualitative conclusions emerge.

(1) Even when interior random coil eyelets are permitted in principle, in practice, their statistical weight is negligible. 
Thus the loops-excluded and interior eyelet models give identical results and the single interacting $\alpha$-helical stretch always contains the cross-linked pair of blocks. This is true provided the statistical weights of the cross-linked and non-cross-linked interacting blocks are the same order of magnitude.

(2) Because of conclusion 1, any differences in initiation and propagation parameters induced by stress at the cross-link alone will to a great extent be subsumed in the configurational volume ratio, $r_{\phi}$. The reasonable range of values of $r_{\phi}$ is on the order of $10^{-1}$ or less.

(3) The experimentally determined overall helix content of the cross-linked dimer, $f_{\text {he }}$, includes contributions from both noninteracting conformations, with helix content $f_{\mathrm{hm}}$, and interacting conformations with helix content $f_{\mathrm{d}}$, the relative contribution being mediated by $r_{\phi}$. Hence the helix-coil transition as measured by $f_{\text {he }}$ will be more cooperative than that for the subpopulation of non-crosslinked dimers containing at least a single interacting pair of $\alpha$-helical turns. This is in qualitative accord with recent work in which theory is applied to experiment on tropomyosin. ${ }^{14,35,37}$

(4) The theory predicts that if the cross-link is placed at a chain end, the helix-coil transition should be much more cooperative than if the cross-link is placed near the middle of the chain. This is entirely a consequence of the continuous nature of the helix-coil transition and is due to the reduction of configurational states accessible to the chains consistent with the constraint that the cross-linked block must be helical. The alternative viewpoint that treats the denaturation of tropomyosin as proceeding via the melting in all-or-none stages of cooperative domains would be hard pressed to rationalize such enhanced cooperativity. ${ }^{40,41,43,44}$ Thus, it is extremely important that studies of the stability of singly cross-linked, two-chain, coiled coils as a function of cross-link location be undertaken. Furthermore, based on the homopolymeric analogy, the theory predicts that the $\beta$-tropomyosin dimer containing a single cross-link at residue 36 or 190 should be quite close in stability. It is the cross-linking of the chain in one of the six or so $\alpha$-helical turns near the chain ends that should produce the most pronounced effect.

Conclusions 1 and 2 imply that unless the perturbation of the statistical weight of the cross-linked interacting helical block pair is so great as to prohibit interacting helical states (in direct contradiction to experiment), the interacting cross-linked block should be very highly helical. If the cross-linkable residues are near the middle of the chain, it should be highly helical whether the cross-link is present or not. Previously, it was reported that based on ESR studies of tropomyosin, the spin-labeled cysteine-190 region of the molecule was believed to be less stable than the molecule as a whole. ${ }^{43,44}$ This is in apparent contradiction to the theory for both cross-linked and non-cross-linked chains. In fact, the more recent work on spin-labeled cysteine-190 19 indicates that the opposite is true. Under the appropriate experimental conditions where the spin label leaves the native backbone chemical structure intact, ESR studies of the cysteine- 190 region indicates limited mobility exists until the temperature regime where the two non-cross-linked chains start to dissociate. Thus, the spin label studies on tropomyosin having the native backbone structure are in agreement with the qualitative predictions presented here.

Conclusions 1 and 2 have an additional implication. Suppose the $\sigma$ and $s$ of the cross-linked block are known. For chains containing a cross-link near the middle of the chain, the true volume ratio $r_{\phi}=u_{\phi} / v_{\phi}$ (without the factor correcting for the effective statistical weight of the crosslinked block) depends explicitly on properties of the cross-link via the relative configurational entropy of the cross-link in the interacting helical state as compared to the noninteracting state. We would expect the orientational factor of the two $\alpha$-helices with and without a cross-link to be the same.

At this point, several avenues of research need to be investigated. The theory of singly cross-linked chains developed here should be applied to tropomyosin and to a synthetic analogue of tropomyosin that contains a cross-link near an end, ${ }^{30}$ which was previously and incorrectly treated by neglecting the presence of the cross-link. ${ }^{45}$ Moreover, the theory should be extended to include the possibility of out-of-register conformations that by necessity must contain constrained interior random coil loops originating at the cross-link. On the basis of our work here, we would expect such out-of-register states to contribute negligibly, but this expectation remains to be verified. Finally, we are in the process of extending the interior eyelet model to doubly cross-linked, two-chain, coiled coils. (In time, it may be possible experimentally to double cross-link $\beta$-tropomyosin.) If the molecule is not completely helical and interacting between cross-links, it must contain closed interior random coil loops that terminate on interacting helical stretches. The loops-excluded model excludes such states a priori and might be invalid when constrained, interior random coil loops are the only allowed random coil states. Once the theory of the helix-coil transition in doubly cross-linked dimers is formulated, the equilibrium theory of the helix-coil transition in two-chain, coiled coils, at this level of development, will be complete.

Note Added in Proof: Recent work indicates that in the doubly cross-linked, homopolymeric analogue of tropomyosin, the helix-coil transition is of an all-or-none character; either the molecule is fully helical and interacting between cross-links or it lacks any interacting pairs of helices whatsoever. ${ }^{46}$

Acknowledgment. This work was supported in part by a grant from the Biophysical Program of the National Science Foundation (Grant No. PCM 82-12404). Thanks are due to C. Skolnick for a critical reading of the manuscript.

\section{References and Notes}

(1) Skolnick, J.; Holtzer, A. Macromolecules 1982, 15, 303.

(2) Skolnick, J. Macromolecules 1983, 16, 1069.

(3) Skolnick, J. Macromolecules 1983, 16, 1763.

(4) Skolnick, J. Macromolecules 1984, 17, 645.

(5) Szent-Gyorgyi, A. G.; Cohen, C.; Kendrick-Jones, J. J. Mol. Biol. 1971, 56, 239.

(6) Cohen, C.; Szent-Gyorgyi, A. G. J. Am. Chem. Soc. 1957, 79, 248.

(7) Cohen, C.; Holmes, K. J. Mol. Biol. 1963, 6, 423.

(8) Lowey, S.; Kucera, J.; Holtzer, A. J. Mol. Biol. 1963, 7, 234.

(9) Holtzer, A.; Clark, R.; Lowey, S. Biochemistry 1965, 4, 2401.

(10) Woods, E. Biochemistry 1969, 8, 4336.

(11) Caspar, D.; Cohen, C.; Langley, W. J. Mol. Biol. 1969, 41, 87.

(12) Hodges, R.; Sodek, J.; Smillie, L.; Jurasek, L. Cold Spring Harbor Symp. Quant. Biol. 1972, 37, 299.

(13) Zimm, B.; Bragg, J. J. Chem. Phys. 1959, 31, 526.

(14) Skolnick, J.; Holtzer, A. Macromolecules, in press.

(15) Yukioka, S.; Noda, I.; Nagasawa, M.; Holtzer, M. E.; Holtzer, A. Macromolecules, in press.

(16) Lehrer, S. J. Mol. Biol. 1978, 118, 209.

(17) Schellman, J. A: C. R. Trav. Lab. Carlsberg, Ser. Chim. 1955, $29,230$.

(18) Flory, P. J. J. Am. Chem. Soc. 1956, 78, 5222.

(19) Jacobson, H.; Stockmayer, W. H. J. Chem. Phys. 1950, 18, 1600.

(20) McLachlan, A.; Stewart, M. J. Biol. Chem. 1975, 98, 293.

(21) Stone, D.; Smillie, L. J. Biol. Chem. 1978, 253, 1137.

(22) Mak, A.; Lewis, W.; Smillie, L. FEBS Lett. 1979, 105, 232. 
(23) Crothers, D.; Kallenbach, N. J. Chem. Phys. 1966, 45, 917.

(24) Poland, D.; Scheraga, H. A. "Theory of Helix-Coil Transitions in Biopolymers"; Academic Press: New York, 1970.

(25) Mattice, W. L.; Skolnick, J. Macromolecules 1982, 15, 1088.

(26) Mattice, W. L.; Srinivasan, G.; Santiago, G. Macromolecules $1980,13,1254$.

(27) Maroun, R.; Mattice, W. Biochim. Biophys. Acta 1984, 784, 133.

(28) Ihara, S.; Ooi, T.; Takahashi, S. Biopolymers 1982, 21, 131.

(29) Mayer, J.; Mayer, M. "Statistical Mechanics"; Wiley: New York, 1940; pp 213-217.

(30) Hodges, R.; Saund, A.; Chong, P.; St. Pierre, S.; Reid, R. J. Biol. Chem. 1981, 256, 1214.

(31) Flory, P. "Statistical Mechanics of Chain Molecules"; Wiley: New York, 1969; p 251.

(32) Schultz, G.; Schirmer, R. "Principles of Protein Structure"; Springer-Verlag: New York, 1979.
(33) Holtzer, M.; Holtzer, A. Macromolecules 1972, 5, 294.

(34) Skolnick, J. Macromolecules, in press.

(35) Skolnick, J.; Holtzer, A., unpublished results.

(36) Krishnan, K. S.; Brandts, J. F.; Lehrer, S. FEBS Lett. 1978, 91, 206.

(37) Holtzer, M. E.; Holtzer, A.; Skolnick, J. Macromolecules 1983, 16,173 .

(38) Pato, M.; Mak, A.; Smillie, L. J. Biol. Chem. 1981, 256, 593

(39) Williams, D. L., Jr.; Swenson, C. Biochemistry 1981, 20, 3856.

(40) Potekhin, S.; Privalov, P. J. Mol. Biol. 1982, 159, 519.

(41) Betteridge, D.; Lehrer, S. J. Mol. Biol. 1983, 167, 481.

(42) Holtzer, M. E.; Holtzer, A.; Skolnick, J. Macromolecules 1983 , 16,462 .

(43) Chao, Y. Y. H.; Holtzer, A. Biochemistry 1975, 14, 2164.

(44) Graceffa, P.; Lehrer, S. Biochemistry 1984, 22, 2606.

(45) Skolnick, J.; Holtzer, A. Macromolecules 1982, 15, 812

(46) Skolnick, J. Macromolecules, submitted.

\title{
Theory of $\alpha$-Helix-to-Random-Coil Transition of Two-Chain, Coiled Coils. Application of the Augmented Theory to Thermal Denaturation of $\alpha$-Tropomyosin
}

\author{
Jeffrey Skolnick ${ }^{\dagger}$ and Alfred Holtzer* \\ Department of Chemistry, Washington University, St. Louis, Missouri 63130. \\ Received November 21, 1984
}

\begin{abstract}
The statistical mechanical theory of the helix-to-random-coil transition in two-chain, $\alpha$-helical coiled coils has recently been augmented by inclusion of the effects of loop entropy and out-of-register ("mismatched") structures. This theory is applied to experimental data on non-cross-linked $\alpha$-tropomyosin at nearly neutral and at acidic $\mathrm{pH}$, using extant values of helix initiation $(\sigma)$ and propagation $(s)$ parameters for each amino acid in the sequence. A semiquantitative fit of the helix content (from circular dichroism measurements) vs. temperature $\left(0-80^{\circ} \mathrm{C}\right)$ is obtained at each $\mathrm{pH}$, covering a 1000 -fold range of protein concentration. The algorithms for the mean interhelix interaction free energy per mole of turn pairs $(R T$ $\ln w(T))$ needed to produce the fit at each $\mathrm{pH}$ provide curves of $R T \ln w(T)$ vs. $T$ that are similar in range and in shape, each showing a minimum near room temperature. Theory is also compared with independent experiments, in particular light scattering and cross-linkability studies at nearly neutral $\mathrm{pH}$. The temperature dependence of the weight-average molecular weight at nearly neutral $\mathrm{pH}$, as recently determined by light scattering, agrees well with the theoretical prediction. The observed high degree of cross-linkability of tropomyosin in the native state can be reconciled with the theoretically calculated fraction of in-register molecules under the benign conditions of the cross-linking experiments. Examination shows that the principal cause of the greater stability of $\alpha$-tropomyosin at low $\mathrm{pH}$ lies in the augmented short-range $(\sigma, s)$ interactions of aspartic and glutamic residues over those of the aspartate and glutamate species which predominate near neutral $\mathrm{pH}$. In fact, it is shown that, with small adjustment (within experimental error) in these parameters, the same interhelix interaction free energy algorithm can be used to explain the full range of data at both pHs. A discussion of the implications of this result is given, wherein it is shown that the interhelix salt bridges, while they may provide enough free energy at nearly neutral $\mathrm{pH}$ to ensure that the helices associate in parallel, make a contribution to the total interhelix interaction that is relatively small compared with the hydrophobic contribution. The statistical theory developed here is compared with the all-or-none-stages model brought forward elsewhere; it is suggested that the latter disagrees with the recent light scattering data and is difficult to reconcile with accepted ideas concerning loop entropy.
\end{abstract}

\section{Introduction}

The native tropomyosin molecule is a two-chain, $\alpha$ helical, coiled coil. ${ }^{1}$ That is, the accepted picture of the molecule is that it comprises two essentially completely $\alpha$-helical polypeptide chains arranged side-by-side, in parallel and in register, and given a slight supertwist. ${ }^{2-8}$ The simplicity of this structure makes it an attractive object of study as much for its relevance to the larger problem of protein conformational stability as for its inherent interest in the biochemistry of muscle. ${ }^{9-14}$ Here is a fully functional protein that has only one type of secondary structure ( $\alpha$-helix), no tertiary structure (i.e., no folding of a given chain back upon itself), and a quaternary structure that allows chain-chain interactions only in a

\footnotetext{
${ }^{+}$Alfred P. Sloan Foundation Fellow.
}

fixed, repetitive geometrical context. The molecule thus forms a natural model system for the problem of sorting out "short-range" and "long-range" contributions to the conformational stability of protein structures and their relationship to the primary structure.

Determination and study of the primary structure of tropomyosin have clarified, at least in a qualitative way, some aspects of the array of stabilizing forces. ${ }^{15-17}$ The amino acid sequence in each 284-residue chain is based on a pseudo-repeating heptet (denoted by letters a-g) in which the first and fourth residues (a, d) are obligatory hydrophobes, the fifth (e) bears an obligatory negative charge, and the seventh $(\mathrm{g})$ bears an obligatory positive charge. When such a chain is coiled into a right-handed $\alpha$-helix, the resulting structure is locally highly amphipathic; the obligatory hydrophobes appear on one face of the resulting molecular cylinder, while a line of positive charge appears 